University of Rhode Island

DigitalCommons@URI

Open Access Dissertations

1991

\title{
Experientially-Modulated Neuronal Death in Overconnected Neural Networks
}

Thomas J. Skrmetti

University of Rhode Island

Follow this and additional works at: https://digitalcommons.uri.edu/oa_diss

\section{Recommended Citation}

Skrmetti, Thomas J., "Experientially-Modulated Neuronal Death in Overconnected Neural Networks" (1991). Open Access Dissertations. Paper 827.

https://digitalcommons.uri.edu/oa_diss/827

This Dissertation is brought to you for free and open access by DigitalCommons@URI. It has been accepted for inclusion in Open Access Dissertations by an authorized administrator of DigitalCommons@URI. For more information, please contact digitalcommons-group@uri.edu. 
EXPERIENTIALLY-MODULATED NEURONAL DEATH

IN OVERCONNECTED NEURAL NETWORKS

BY

THOMAS J. SKRMETTI

A DISSERTATION SUBMITTED IN PARTIAL FULFILLMENT OF THE REQUIREMENTS FOR THE DEGREE OF

DOCTOR OF PHILOSOPHY

IN

PSYCHOLOGY

UNIVERSITY OF RHODE ISLAND

1991 
DOCTOR OF PHILOSOPHY DISSERTATION

OF

THOMAS J. SKRMETTI

APPROVED :

Dissertation Committee

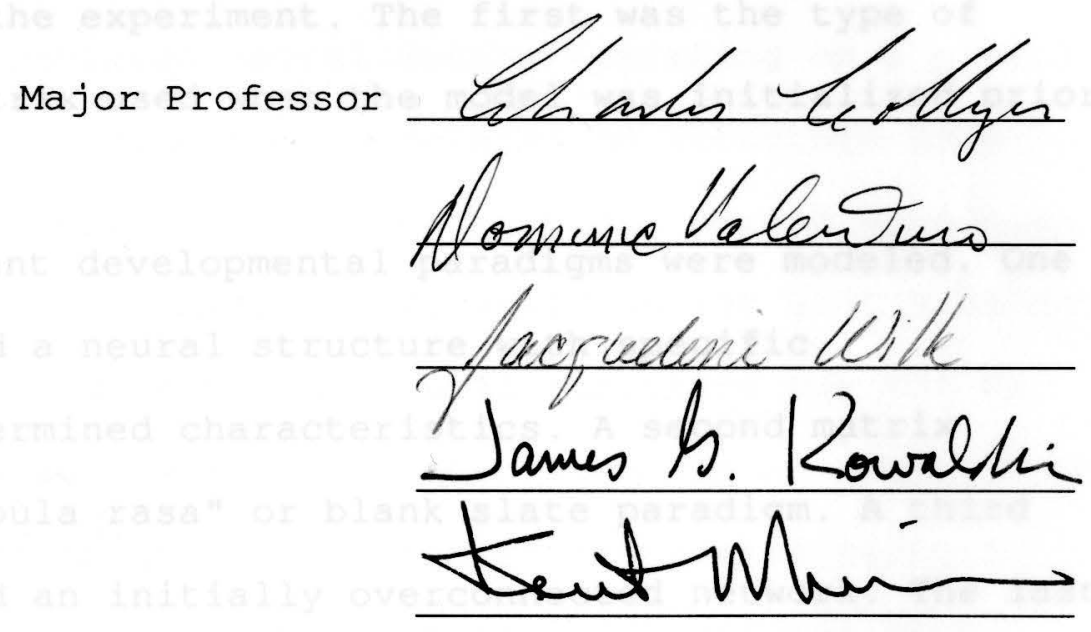

DEAN OF THE GRADUATE SCHOOL 


\section{ABSTRACT}

This experiment was designed to examine the possible physiological mechanisms underlying the formation of critical periods in development. It was hypothesized that experientially-modulated neuronal death, coupled with an overconnected neural network, and acting early in the developmental sequence, could produce a learning effect similar to that described as a "critical period effect."

A computer-based associative memory model was used to simulate a simple neural network representing the associative cortex. Three different independent variables were manipulated in the experiment. The first was the type of connectivity matrix used when the model was initialized prior to training.

Four different developmental paradigms were modeled. One matrix simulated a neural structure with specific, genetically-determined characteristics. A second matrix simulated a "tabula rasa" or blank slate paradigm. A third matrix simulated an initially overconnected network. The last was a randomly generated connectivity matrix that reflected no particular paradigm, used as a control.

A second independent variable was the experiential sequence in which the model was trained. Each developmental model was trained on four different stimulus sets simulating different experiential sequences. 
The third independent variable was the presence or absence of an experientially-modulated neuronal death component. All developmental models were trained across all experiential sequences with and without the neural death component acting on the model.

The model's behavior was assessed using 2 dependent variables - the ability of the model to learn later in development, and the structural fit of the model to the prototype stimulus set. In addition, the model's output was examined for evidence of a critical period effect.

The results of the experiment indicate that a critical period effect can be induced simply by the use of an experientially-modulated neural death, operating on a plastic neural structure. A genetically-determined structure is not necessary for the manifestation of critical period phenomena. All models performed similarly. It would appear that a hardwired, genetically-determined neural structure may not be the optimum paradigm to support later learning in a noisy environment, that is, one where the environmental stimuli differ in some degree from those that the model has been structured to deal with.

Surprisingly, both the ability to learn and the structural fit of the overconnected and experiential models were significantly better than that of the genetic model in noisy environments. Only the randomly-connected model performed worse than the genetic model. It appears that genetically specifying a neural structure that must respond 
to a noisy environment produces problems similar to those of overfitting an analytical model.

The ability of all models to learn a specific stimulus was expected to increase as exposure to the stimulus increased. This behavior was, in fact, observed for all models.

The findings of this experiment indicate the formation of critical periods may be the natural consequence of rapidly removing unused neural pathways early in development, while stabilizing the pathways that are used. If the organism is not exposed to certain types of stimuli, the neural pathways associated with processing those stimuli are lost. If the organism is exposed to those stimuli early in development, the circuits are stabilized, and are available for learning later in development. 


\section{ACKNOWLEDGEMENTS}

There are a great many people who have supported me in this undertaking and I would like to thank them for their help.

I would first like to thank my wife, Susan, not only for her continued support and encouragement, but also for recognizing, so many years ago, a "lifelong learner" in such an unlikely candidate.

I would like to thank my children, Jonathan and Sarah, for their unfailing enthusiasm for my studies. They have lived their entire lives with their father as a student, have been my most ardent supporters, and are not yet aware of the extent of their contribution to my work.

I would like to thank the members of my committee, Dr. Dominic Valentino, Dr. Jacqueline Wilk, and Dr. James Kowalski for their interest and suggestions during the formulation and execution of this research. I would also like to thank my dear friend and colleague, Dr. Carol Davis for her time, effort and support in all facets of my work.

I would particularly like to thank my major professor, Dr. Charles Collyer for providing me with his scholarly advice and wisdom, while allowing me the freedom to pursue my own course. His unfailing support, trust, and friendship will always be valued.

This dissertation is dedicated to Louise Mallard. 


\section{PREFACE}

There were 2 main reasons for performing this specific study. The first involved the investigation of a specific research question. Neuronal death has long been recognized to be an important factor in the early development of an organism. Its exact role, however, has never been fully defined. By linking the opposing mechanisms of neuronal death and neural stabilization, the experimenter hoped to demonstrate that neuronal death, modulated by experiential history, is an effective sculptor of neural structure that allows an organism to be born into a wide variety of possible environments and yet quickly adapt to successfully exploit, and efficiently function in, a specific environment.

The findings of this experiment are applicable to a wide range of psychological research. They provide insight into a possible physiological mechanism to explain the effects of early deprivation on later development, and suggest an area of inquiry regarding early intervention techniques. In addition, the experiment has provided evidence of a possible physiologically-based linkage between learning paradigms and behavioral, developmental, and cognitive paradigms. As such, the results of this experiment should be of value to developmental, educational, and behavioral psychologists.

The second reason for pursuing this experiment involves a broadening of the current theoretical perspectives that seem to be prominent in many areas of psychological research. It 
is widely accepted by a large number of psychologists that the days of the grand theories are over. Indeed, many psychologists do not even think that such theories are needed, and that theories of a more limited scope are sufficient for psychology to continue to progress, even to thrive. It is perhaps time to reassess this position.

In the past 20 years, there have been great strides made in linking the findings of physiological psychology with the fields of behavioral, developmental, and cognitive psychology. Psychology is rapidly approaching the point where a comprehensive theory linking all of the diverse areas of psychology may be possible. To realize this end requires the design and execution of a wide range of experiments that attempt to integrate findings from many psychological fields in order to derive models which exhibit behaviors or characteristics that have been accepted as important components of other areas of psychology.

The experiment described in this paper is an attempt to expand on the scope of such studies and to help provide a base of experimental work that shows that such a linkage is not only desirable, but necessary for psychological research to achieve its full potential. 


\section{TABLE OF CONTENTS}

Page

ABSTRACT .................... ii

ACKNOWLEDGEMENTS................... . v

PREFACE.........................

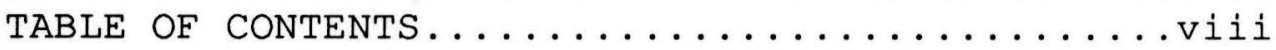

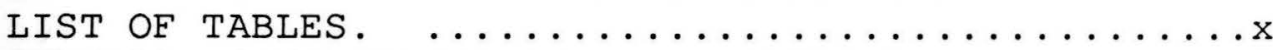

LIST OF FIGURES.......................

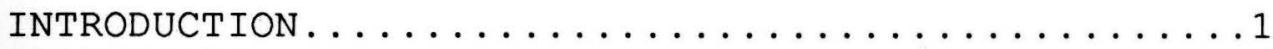

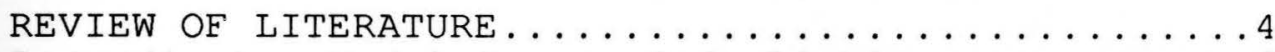

Critical Periods.....................

Overconnected Networks and Neural Death........6

Mechanisms of Experiential Change.............

Genetic versus Generic Structures..............10

Summary ............................. 12

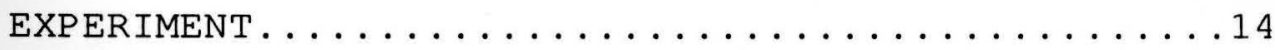

Introduction. ....................... 14

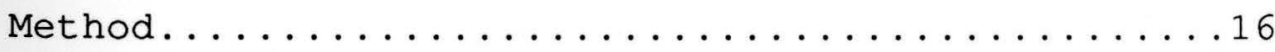

Characteristics of the Model..............16

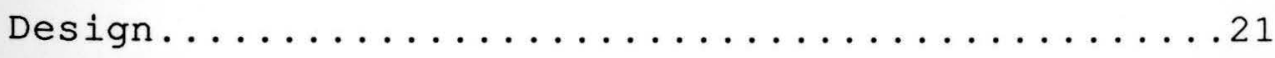

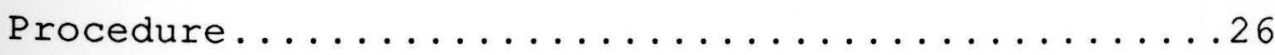

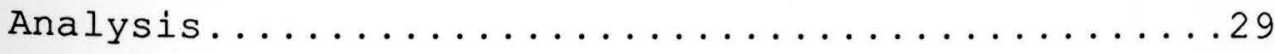

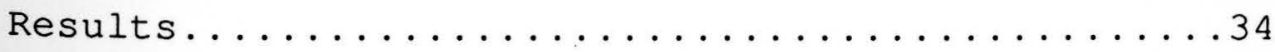

Summary .............................. 34

Number of Required Learning Cycles.............38

Sum-Squared Error............................... 


\section{Page}

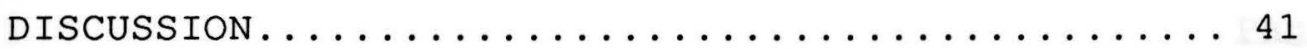

Critical Period Effect................ 42

Model Type..........................42

Environmental Sequence................48

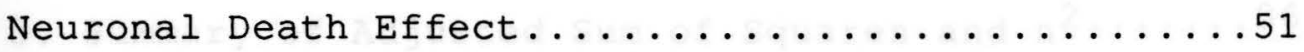

SUMMARY, CONCLUSIONS AND IMPLICATIONS.........55

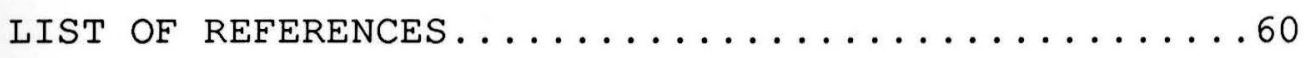

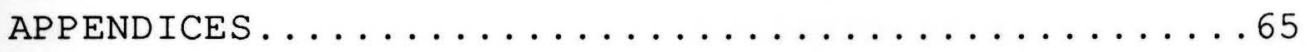

Appendix A - Model Source Code Listing.........665

Appendix B - Stimulus Data Sets..............81

Appendix C - Summary of Means..............82

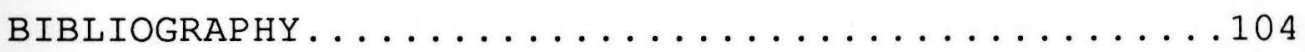


1. Summary of Means and Standard Deviations......84

2. Summary of MANOVA Results...............85

3. Summary of Adjusted Sum of Squares and $n^{2} \ldots \ldots 86$

4. Summary of Univariate Analyses.............87 


\section{LIST OF FIGURES}

Page

1. Network Architecture.....................88

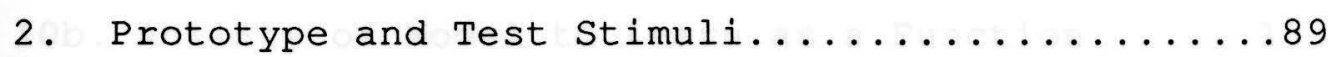

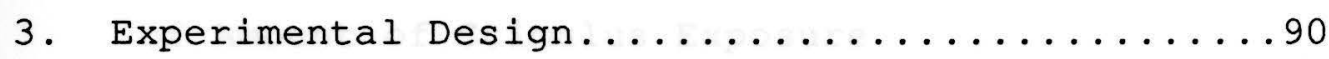

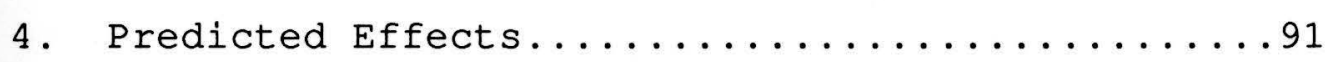

5. Performance of Model as a Function of.........93 Environmental Sequence

6a. Generic Model's Ability to Learn as a.........94 Function of Environmental Sequence

6b. Experiential Model's Ability to Learn.........95 as a Function of Environmental Sequence

6c. Overconnected Model's Ability to Learn.........96 as a Function of Environmental Sequence

6d. Random Model's Ability to Learn as a.........97 Function of Environmental Sequence

7a. Structural Adaptation as a Function of........98 Model Type

7b. Structural Adaptation as a Function of........99 Model Type (By Increasing Error)

8. Structural Adaptation as a Function of........100 Model Type (No Noise in Training Set)

9a. Genetic Model's Performance as a Function......101 of Environmental Sequence (No noise in Training set) 
Page

10a. Structural Fit of Models as a Function of.....102 Amount of Stimulus Exposure

10b. Ability of Model to Learn as a Function.......103 of Amount of Stimulus Exposure 
EXPERIENTIALLY-MODULATED NEURONAL DEATH IN OVERCONNECTED NEURAL NETWORKS 


\section{INTRODUCTION}

There has been a long-standing debate on the relative contribution of nature and nurture in human psychological development (Lerner, 1986). Stage theorists attribute development to a maturational "unfolding," driven, at least in part, by genetically-programmed characteristics inherent in the organism. Empiricists, on the other hand, attribute development to an essentially continuous, quantitative process, driven by the cumulative effects of experiences. Interactionists combine the 2 perspectives, attributing development to the interaction of genetic and experiential factors.

The study of critical periods is a subset of the study of more general developmental phenomena. The arguments and theories about the underlying mechanisms of critical period phenomena closely parallel the more general nature-nurture arguments usually associated with overall human development. Critical period phenomena provide a well-bounded problem space in which to study key aspects of genetic and environmental contributions to overall development.

Detailed experimentation involving the relative contributions of genetics compared to environment would, ideally, require a population consisting of several groups of genotypes. Within each group, all members would share identical genotypes and experiential histories. Members of each group would then be subjected to different experiential sequences, and the relative impact of genetics and 
environment could be experimentally assessed. Such a population is, obviously, not available. Even if such a subject pool was available, the ethical problems involved in manipulating experiential histories would restrict such experimentation to simple manipulations.

Associative memory models are computer-based models that simulate basic neural circuits. These models allow a researcher to simulate variations in physiological factors such as initial (genetic) connectivity of neural structures, and to simulate theoretical models of experiential change on the structure and functioning of these circuits. In addition, the same model can be repeatedly initialized in a given state, and subjected to any number of experiential sequences. Thus, these models can, in effect, provide a limitless population of subjects with identical "genotypes." As such, associative memory models provide researchers with a powerful tool to examine developmental issues.

This experiment uses associative memory models to represent different developmental paradigms - the genetic, the experiential, and the interactionist. These models incorporate special functions to simulate recent physiological findings involving experientially-modulated neuronal death, and the experiential stabilization of neural structures. The models representing these paradigms are trained in different sequences to simulate the effects of differing experiential histories. 
The output of the models is then examined to assess the relative contribution of initial structure and experiential history to the model's overall ability to learn, and to the formation of critical period effects. 


\section{REVIEW OF THE LITERATURE}

\section{Critical Periods}

Critical (or sensitive) periods of development are times in the developmental cycle when an organism is especially susceptible to the developmental influences of experience (Flavell, 1985; Munro, 1986). An excellent example of this phenomena is song learning in some species of birds (Schwartz, 1989).

If some species of birds are exposed to their speciesspecific song, even briefly early in life, they will be able to successfully reproduce the song at maturity. This effect has been found to occur even if they are denied exposure to the song for the remainder of their development. If, however, the birds are not exposed to the species-specific song at a specific time early in development, they will be unable to learn the song later in life, even with frequent exposure. Many other examples of critical period phenomena have been described including the development of ocular-dominance columns in kittens, language development in children, and experientially-induced changes in the cortical structure of kittens (Spinelli and Jensen, 1979).

It is as if there is a "window of susceptibility" in some species where the organism is especially sensitive to certain types of environmental stimuli. If the organism is exposed to the appropriate stimuli, a significant change occurs in the organism's neural structure. If the organism fails to 
encounter these stimuli early on, the "window of susceptibility" closes, and later development is hindered. Hypotheses attempting to explain critical or sensitive period phenomena have described a number of widely differing mechanisms. Lorenz proposed a purely biological explanation of these phenomena (Lerner, 1986). In this view, the basis of the critical period phenomena observed in organisms was strictly due to a maturational timetable driven by genetically-controlled processes inherent in the developing organism. Supporting evidence for the biological perspective includes imprinting (Catania, 1984), song learning in birds (Schwartz, 1989), and language acquisition in children (Restak, 1988; Stillings, et al. 1989).

Munro (1986) has described an almost opposite mechanism where the phenomena of critical periods may be the result of experiential change operating at the level of the individual neuron. The experiential component has found support through the work performed by Hubel and Wiesel (Carlson, 1986; Munro, 1986; Squire, 1987). More recently, Cynader manipulated the timing of critical periods in kitten vision through the use of dark-rearing techniques (Aoki and Siekevitz, 1988), and Stryker and Harris manipulated critical periods through the use of a neurotoxin which prevented action potentials in the retina of kittens (cited in Kalil, 1989).

Many current developmentalists propose an interactionist mechanism to account for sensitive periods (Lerner, 1986). In this view, the organism is more susceptible to certain 
influences early in life, but, if these influences are not present, the organism can learn related stimuli later in life, although less efficiently than when exposed at an early age.

This interactionist viewpoint places differing emphasis on maturational or experiential factors at different times in an organism's development. More importantly (from the standpoint of this hypothesis), this perspective emphasizes the interaction of these contributions.

\section{Overconnected Networks and Neuronal Death}

It has been noted that many animals possess many more neurons early in the development cycle than at maturity (Purves and Lichtman, 1980; Rakic and Riley, 1983; Squire, 1987; Restak, 1988). In the adult, however, there are a greater number of synapses associated with each neuron. In fact, although the overall number of neurons is drastically decreased in the adult, the overall number of synapses may actually increase (Lichtman, 1978; Purves and Lichtman, 1980). These findings lead to the supposition that there are at least two mechanisms operating to regulate the neural structure in developing organisms. The first involves a pruning of available pathways or linkages. A second mechanism involves some type of reinforcement or expansion of the surviving pathways.

Alkon, in his work on classical conditioning (1987, 1989), has observed just such changes in the neural system 
that are attributed to the learning process. These changes include both the strengthening of active neural connections, and the pruning of unused neural pathways.

The strengthening of used pathways and the pruning of unused pathways has been termed "competitive learning." It is as if neurons "compete" for resources, and that the more active a neuron is, the larger its share of those resources will be (Purves and Lichtman, 1980).

Gerald Edelman has described this competitive process as "neural Darwinism" (Smoliar, 1989). Neurons and neuronal pathways that most reflect the experiential environment of the organism tend to be activated more than those that do not reflect the immediate experiential environment. This phenomenon shapes an organism's neural structure, so that, over time, the neural structure tends to correlate with the organism's experiential environment. That is, the neural structure "adapts" to the organism's environment.

\section{Mechanisms of Experiential Change}

Donald Hebb (as cited in Squire, 1987) proposed an experiential theory of learning which involved a reduction in the synaptic resistance between 2 associated neurons. When 2 neurons in close proximity fired together, the resistance between them was decreased. Therefore, in the future, when one of the 2 neurons fired for whatever reason, the second neuron also tended to fire, due to the reduced resistance. Thus, any associations between the 2 neurons were 
strengthened. Experience, in Hebb's view, was an active shaper of the overall structure of the nervous system.

Brown and his colleagues (1988) describe a form of synaptic change which is the result of pre and post-synaptic structural changes. This process, long-term potentiation (LTP) is dependent on temporal contiguity of the pre and post-synaptic events. If the pre-synaptic neuron experiences an action potential at about the same time as the postsynaptic neuron is depolarized by another input, a rapid, long lasting synaptic enhancement will result between the two neurons. That is, further activity in the pre-synaptic neuron will tend to more easily excite the post-synaptic neuron. Contiguous presentation of two stimuli has resulted in an association (or at least the start of an association) between the pre-synaptic stimulus and the post-synaptic response which closely resembles the effects posited by Hebb.

Lynch and Baudry have observed physiological changes in neural structure that can be linked directly to experience. When a neuronal membrane becomes depolarized after an action potential, $\mathrm{Ca}^{++}$ions flowing into the cell activate calpain molecules. The calpain molecules interact with fodrin molecules lining the neuronal membrane. Calpain causes cleaving of the fodrin molecules which, in turn, expose glutamate receptors (Carlson, 1986; Squire, 1987).

The more the neuronal membrane is stimulated, the more fodrin is cleaved and the more glutamate receptors are exposed. Since the sensitivity of a neuron is (at least in 
part) proportional to the number of receptors, the sensitivity of the neuron increases, effectively reducing the membrane's resistance. Thus the calpain / fodrin reaction provides a possible physiological mechanism which closely resembles a form of Hebbian learning.

An even more fundamental effect of experience on neural structure is described by Aoki and Siekovitz (1988). This effect is the phosphorylization of the microtubule-associated protein 2 (MAP-2) molecule, a molecule that is contained in all neurons. This molecule controls the actual structure of the neuron by controlling the shape of the micro-tubules making up the neuron's internal structure. MAP-2 molecules are de-phosphorylated as a result of action potentials occurring in the neuron. When the MAP-2 molecules are dephosphorylated, the neuronal structure is fixed. If the molecules are fully phosphorylated, the neuronal structure is malleable and plastic.

The MAP-2 effect appears to be a form of non-associative change. MAP-2 effects can, however, have significant impact on associative processes. In both Brown et. al's work on LTP and Alkon's work on classical conditioning, a synaptic connection between the two neurons is required to exist to support further constructive changes. Therefore, a key element necessary to support associative conditioning appears to be the availability of intersecting neural circuits supporting processing of the CS and the US. 
Once MAP-2 molecules are de-phosphorylated by a succession of action potentials, the neural structure is basically fixed. If the neuron does not undergo any further action potentials, the MAP-2 molecules will slowly phosphorylate, and the neural structure will again become plastic. Aoki has found, however, that even the baseline firing rate of a neuron is enough to cause significant dephosphorylization of the MAP-2 molecule. Therefore, once the neural structure is established, it is unlikely to change significantly.

Since the neural structure is now fixed, associative learning can only take place between circuits that share common connection points. The degree of connectedness therefore sets limits on the amount of conditioning that can occur between groups of neurons.

\section{Genetic versus Generic Neural Structures}

Learning in higher level mammals (i.e., man), occurs on a scope and time scale that precludes structural modification by the genetically-driven processes of evolution. It may be that mammals have evolved a mechanism that allows for rapid adaptation to a given environment with minimal evolutionary investment.

The neocortex is the largest structure in the mammalian brain. In modern man, the cortex and its connections occupy $80 \%$ of the brain volume (Passingham as reported in Douglas and Martin, 1990). 
There are 3 different types of cortex: koniocortex (e.g., the primary visual system); agranular cortex (e.g., motor cortex); and associative cortex. All cortical areas are composed of distinct layers, made of different types and densities of neurons.

The basic organization of these cortical layers are vertical "columns" of neurons arranged perpendicularly to the surface of the cortex. These columns contain between 100 to 1000 neurons, and are surprisingly uniform across all types of neocortex (Squire, 1987; Douglas and Martin, 1990). According to Mountcastle, (as cited in Squire, 1987) "... the processing function of neocortical modules is qualitatively similar in all neocortical regions."

As stated by Douglas and Martin (1990), "That the neocortex has expanded so rapidly suggests that it performs its functions extremely well, that its wiring is easy to replicate, and that further expansion requires few genetic instructions."

Neurons in the cortex have been shown to be highly plastic and sensitive to experiential change. Thus, cortical columns provide a generic, undifferentiated, neural structure that is capable of adapting to varying experiential environments. 


\section{Summary}

Genetically-tuned physiological structures take enormous amounts of time to evolve. Because of the high evolutionary cost in time, it can be expected that, if these structures have, in fact evolved, they would likely not be plastic, or sensitive to environmental change. There is no logic to spending eons tuning a fine-grained structure, then allowing that structure to be modified by the environment. There is a great deal of logic, however, in developing a more generic structure that can provide a malleable substrate that can easily be matched to suit a given environment.

Nature would only have to provide a structure roughly suited to gross environmental conditions. The environment that the organism is born into would then rapidly shape the neural structure of the organism to correlate closely with its actual micro-environment. An initially overconnected neural network, coupled with mechanisms of rapid, experientially-modulated neuronal death, and stabilization provide a possible mechanism to accomplish this.

A peculiar outcome of rapid, experientially-modulated death and stabilization should be an effect similar to that described in developmental literature as a critical period. If the organism encounters the type of stimuli that will stabilize critical neural circuits early in development, those circuits will be available to support later learning. If, however, the organism is not exposed to those stimuli, the neural circuits are pruned out, and there is no structure 
available to support later learning of the type represented by that particular type of stimuli.

It is important to note that these mechanisms are proposed to operate only at the highest levels of brain organization, that is, the neocortex of the mammalian nervous system. This part of the brain is assumed to be a recently evolved processing system with a minimal level of genetically-programmed structure. Experiential history is thought to be the critical factor in determining its ultimate microstructure.

There may be other mechanisms acting in the lower areas of the brain (e.g., cerebellum) which can produce critical period effects due to other causes. The effects investigated in this experiment, however, examine functions and structures associated with the cortical regions of the mammalian brain. 


\section{EXPERIMENT}

\section{Introduction}

This experiment was designed to examine how simulated biological and experiential factors interact to allow an organism to most efficiently adapt to a wide range of potential environments. The experiment centered around a computer model which incorporated mechanisms representing the biological contribution to the initial structure of the nervous system and the effects of structural change brought about through the processes of neuronal death and experientially-driven, synaptic change.

It was hypothesized that the overconnected neural networks found in the early stages of development may provide the substrate on which the broadest possible combination of experiences can be assessed. That is, rather than try to hardwire a complex system such as the brain to suit a particular environment (a natural counterpart to overfitting a model), nature may have provided these overconnected brains which already contain the wiring able to adapt to a wide variety of environments (the natural counterpart to a generalizable model). Unused pathways are quickly pruned as the organism develops, through the process of neuronal death. Pathways that are needed to represent and deal with the organism's environment are stabilized and fine-tuned through structural modification processes such as the MAP2 effect and Long-Term Potentiation. 
The basic premise is similar to the story where a woodcarver was asked to explain how he carved such lifelike duck decoys out of a simple block of wood. The woodcarver replied, "I just start whittling and whittle away anything that doesn't look like a duck." Likewise, nature might use the antagonistic forces of neuronal death and experiential change to rapidly prune unneeded connections from an overconnected network to transform it into a form suitably adapted to a particular environment. After the initial substrate has been molded to conform to the overall environment, constructive "tuning" processes which modify the synaptic connectivity between surviving neuronal pathways allow the organism to fine tune this structure.

Because of the rapid reduction of potential circuits occurring as a result of neuronal death in the early stages of development, an organism tends to have periods early in life where certain experiences can have a great impact on the resulting structure of the neural systems. This impact may result in changes in neuronal plasticity due to the stabilizing influence of processes such as those associated with the MAP2 molecule, and may negate the mechanisms responsible for rapid neuronal death.

Following this stabilization (the result of experientially-modulated pruning), the organism would possess a neural structure grossly tuned to fit its particular environment. This is similar to prototype or concept formation as discussed by Knapp and Anderson (1984) where a 
neural model initially extracts prototypes from a set of related stimuli. After they are established, these pathways can then be fine-tuned to the environment through constructive learning processes such as Long Term Potentiation (Squire, 1987). This would represent a model being able to learn specific exemplars from a set of related stimuli.

If, however, these experiences do not occur before the basic circuits have been eliminated, the critical connections will not be available for stabilization or subsequent modification. Therefore, the opportunity for experiential change in these circuits will have been lost.

The end result would be an effect similar to that described as a critical or sensitive period in development. Experience provided before a certain point would enhance an organism's ability to acquire certain behaviors in the future (due to the stabilizing effects of experience on the neuronal structures), whereas lack of those same experiences would tend to reduce the organism's ability to acquire those behaviors (due to pruning of the potential circuits through neuronal death).

\section{Method}

\section{Characteristics of the Model}

The model used in this experiment is an adaptation of a simple linear associator (Hinton and Anderson, 1981; Knapp and Anderson, 1984). Figure 1 illustrates the basic architecture of the model. A simple associator was selected 
for use in these models since the pruning required to simulate neuronal death in the network could introduce complications in interpreting changes in the connection weight matrix when used with more complex learning algorithms, such as back-propagation.

The model consists of a 9 node input layer (vector), and a 9 node output layer connected via a connection weight matrix. The input vector was used to represent environmental stimuli. The output vector was used to represent how well the model responded to a particular input stimulus. The model's output was calculated as a function of each element of the input vector multiplied by the corresponding connection weight matrix entry in each row of the matrix. The resulting products were then summed across each column of the connection weight matrix to produce the output vector (see Figure 1 and "Procedure Compute_Output_Vector," Appendix A Model Source Code Listing). The output values were clipped as necessary to limit them to a value between 0 and 1 (see Procedure Check_Learning of Appendix A) .

The connection weight matrix entries were modified through a simple, Hebbian-like learning algorithm where activity between 2 neurons strengthen the connection between the neurons. If the input vector entry equaled 1 and the corresponding output vector entry was less than 1, the connection weight matrix entries in the row corresponding to the output vector entry were increased by a factor equal to the corresponding learning rate matrix entry. If, however, 
the input entry vector equaled 0 and the corresponding output vector entry was greater than 0 , the connection weight matrix entries in the row corresponding to the output vector entry were decreased by a factor equal to the corresponding learning rate matrix entry (see Procedure Update_Conn_Mat_Weights, Appendix A).

It is important to note that in most associative memory models, a single node in the input or output vectors is assumed to represent the activity of individual neurons in the nervous system. Therefore, the connectivity matrix linking the input vector to the output vector is assumed to represent the actual connection pattern among those individual neurons.

In this experiment, however, the input and output vectors were meant to represent the cumulative effects of groups of neurons (e.g., a given specialized area of the brain) rather than particular neurons in the brain. The intent was to model the overall behavior of large, complex, integrated areas of the brain, rather than the specific behavior of a small number of brain cells. Thus, in this case, the connection weight matrix was not meant to represent individual neuronal connectivity, but, rather, the cumulative connectivity between large groups of associative neurons.

The model incorporated a pruning algorithm to simulate the effects of experientially-modulated neuronal death on the neural structure as described by Purves and Lichtman (1980). This algorithm reduced the neural connection strengths on 
each learning cycle for those neurons that had not been stimulated by environmental inputs.

The neuronal death algorithm was designed to function with two separate matrices, the stability matrix and the learning rate matrix (see variable declaration, Appendix A) . The stability matrix was intended to reflect the stabilizing nature of experiential effects on the nervous system similar to those described for the MAP2 molecule (Aoki and Siekevitz, 1988). The stabilizing effect (or some similar experiential effect) was hypothesized to modulate the effects of neuronal death.

The model took this stabilizing effect into account in the neuronal death algorithm. If a neuron received stimulation during a training cycle (that is, if the input vector entry equaled 1 and the desired output vector entry equaled 1 for any stimulus presentation) a non-zero entry was made in the corresponding stabilization matrix cell. For example, if input vector element $2=1$ and output vector element $4=1$, then stability matrix entry 2,4=1 (see Procedure Update_Conn_Mat_Weights, Appendix A).

After each complete learning cycle (all training patterns presented), the neuronal death procedure (Procedure Kill_neurons, Appendix A) iterated through all stability matrix entries. If an entry equaled 0 , the neural death algorithm decreased the corresponding learning rate matrix entry by a factor of half the current entry value. If the entry was non-zero (the neuron was stimulated during the last 
learning cycle), the corresponding learning rate matrix entry was left at the current value.

It should be noted that the overall plasticity of a neural circuit can be modulated by changing either the number of neurons in the circuit (global change), or by changing the synaptic density of the neurons present in the circuit (local change). The model used in this experiment applies both of these techniques at various times in the learning phase.

The end result of the neuronal death algorithm was a decrease in effective learning rate for those groups of neurons that did not undergo experiential stimulation. This was equivalent to an overall decrease in plasticity for a group of neurons due to a reduction in the number of neurons present, rather than a decrease in the learning rate of individual neurons.

In the early learning phase, the connection weight matrix was modulated in conjunction with the stability matrix entry to simulate the effects of a decrease in plasticity due to rapid neuronal death. In addition, the connection weight matrix was also modulated by the learning algorithm to simulate the effects of experiential learning due to modifications occuring on the synaptic level similar to those proposed by Hebb.

In the later learning phase, the neuronal death algorithm was turned off and any connection weight changes were due simply to effects associated with Hebbian learning. Thus, a single connection weight matrix was used to represent the 
experiential effects on connectivity due to both global and local changes in neural plasticity. Use of the neuronal death algorithm in the present experimental paradigm is fully described in the procedure section below.

\section{Design}

The experiment was conducted using a computer-based associative memory model to compare the effects of different levels of three independent variables on two dependent variables in a $4 \times 2 \times 4$ factorial design.

Independent Variables. There were three independent variables used in this experiment. The first independent variable was Initialization and involved the method of initializing the connection weight matrix used in the models. There were four levels of this variable (see Figure 1).

Insert Figure 1 about here...

The first level reflected a nativistic or organismic perspective as described by Lorenz (Lerner, 1986) whereby the critical period is thought to be the result of decreasing plasticity of the neural networks due to maturation of a genetically-specified structure. This genetically-determined neural structure was represented by an initial connection weight matrix that was initialized to correspond to the 
connectivity patterns necessary to represent the prototype patterns (see description in Procedure below).

To simulate the genetically-determined decrease in plasticity posited for the organismic perspective, the model incorporated a function that prevented the model from undergoing any weight changes after the initial critical period (see Procedure in Appendix A - Update_Connection Weight_Strengths). This function simulated a critical (versus sensitive) period effect, and represents a complete loss of plasticity in the neural circuits. If the organism is exposed to a type of stimulus at an early period, it can effectively learn related stimuli later in life. If not, the genetically-driven decrease in plasticity prevents learning new stimuli at a later date.

The second level of this variable represented an experiential perspective in line with that described by the classical empiricists (Lerner, 1986). In this network, all changes in the connectivity structure were due strictly to experiential effects. This network was initialized with all connection strengths at zero.

According to a critical period hypothesis proposed by Munro (1986), the observed decrease in plasticity attributed to experiential effects is not actually due to any specific changes in the nervous system, but instead, to the continual reduction in the error signal between the environmental stimuli and the organism's responses as the organism adapts to its environment. 
This hypothesis is strongly reminiscent of the RescorlaWagner model of conditioning (Bolles, 1979). In this model, the amount of conditioning that can occur on any given trial is limited by the amount of conditioning that has already occurred. The overall effect of such a model would be a net decrease in observed plasticity. As such, it provides an interesting link between classical behaviorist theory, and more recent work on computer-based models of distributed, associative memory systems.

The third level reflected the interactionist perspective described by Lerner (1986) which incorporated components representing both biologically-determined characteristics and experientially-driven structural effects. This network was initialized in an "overconnected" state (all of the cells in the matrix were connected at some level of connection weight strength) to reflect the overabundance of neurons found in the early stages of development (Purves and Lichtman, 1980; Rakic and Riley, 1983; Squire, 1987; Restak, 1988).

The fourth level was a variation of the interactionist model where the values of the connection weight matrix are randomly assigned at initialization. This model was included to assess the actual contribution, or utility, of overconnecting the initial neural substrate by comparing the effects of overconnection with those of random connection.

A second independent variable was used to assess the effects of the neuronal death algorithm on the model's behavior. There were two levels of this variable, indicating 
the presence or absence of the neuronal death algorithm in the model.

The third independent variable modeled differential environmental effects. This variable was used to examine the effects of different environments on the emergence of sensitive periods. The environmental variable was manipulated by varying the content and presentation sequence of a series of stimulus patterns that were designed to represent differing experiential environments. There were four levels of this variable represented by different stimulus sets (see Figure 2).

Insert Figure 2 about here...

The first level (or stimulus set) represented the environment where the organism is totally deprived of exposure to the stimuli of interest. It consisted of a set of random stimuli that were not at all associated with the stimuli of interest.

The second set represented the environment where the organism received adequate exposure to the critical stimuli early enough in the developmental period to stabilize the neural circuits required to support later associations, even though these stimuli might be lacking in the subsequent environment. This set presented prototype representations of the stimuli of interest only during the first half of the 
training phase, and a set of random stimuli during the second half of the training phase.

The third set represented an environment where the organism did not receive adequate exposure to the critical stimuli until late in development, after the critical connections are likely to have been pruned through neuronal death. This set presented the prototype stimuli only during the second half of the training cycles.

The last set represented the environment where the organism was continually exposed to the critical stimuli through all phases of development. This set presented the prototype stimuli on all training cycles.

There were two dependent variables. The first was the number of training cycles required for the model to correctly learn to classify a test pattern set. The second dependent variable was the sum-squared error between the input pattern presented to the model and the output pattern with which the model responds, measured at the start of the final training phase of each run to assess the effect of previous exposure to the training stimuli on the overall model structure.

Sample size. The computer models used in this experiment were all deterministic models which will, with the exception of those using a randomized connection weight matrix, exactly reproduce the same output each time the model is run. Because the deterministic models will not exhibit any within-group variance, these models incorporated a random noise function to distort the prototype patterns on each training run. This 
was done using the technique described by Knapp and Anderson (1984) where a prototype stimulus was systematically distorted to provide a set of related exemplars (see Figure 3).

Insert Figure 3 about here...

The model was run ten times for each combination of independent variables. This sample size satisfied the requirement that the number of samples per cell in a MANOVA be greater than the number of dependent variables (Tabachnick and Fidell, 1983).

\section{Procedure}

The connectivity matrix of the genetically-determined (Lorenz) model was initially configured to represent the prototype patterns (see Figure 2). The connection weight matrix values were selected by training the experiential model on the prototype patterns and using the connection weight matrix values found in that model when it had learned to correctly classify the prototype pattern set. This represented the genetically-specified component posited by the organismic developmentalists such as Lorenz (Lerner, 1986; Schwartz, 1989).

The experiential (Munro) model was initialized with all connections in a zero state. This represented the "tabula rasa" or "blank slate" of the newly-formed mind as described 
by Locke and representatives of the empiricist perspective (MacLeod, 1975; Lerner, 1986).

The interactionist model was initialized with a connection weight matrix that initially connected all input elements to all output elements with a value which was equal to twice the average of the connection weight strengths used in the Lorenz model. This gave an overconnectedness ratio of approximately two to one, and represented the overconnectedness cited by Purves and Lichtman (1980), Rakic and Riley (1983), Squire (1987), and Restak (1988).

The fourth model was initialized with a connection weight matrix that connected all input elements to all output elements with a random value, normally distributed between 0.5 and -0.5 .

Following initialization, each model was presented with four different sequences of training and control stimuli to simulate the impact of different environmental conditions on development (see Figure 3 ). The training set was made up of three control and three prototype stimuli. The control stimuli were a randomly selected set of patterns. The training stimuli were a set of patterns that were distortions of the prototypes and were similar to those used by Knapp and Anderson (1984) to investigate category learning. Following training, the model was tested using a set of test stimuli made up of the actual prototype patterns.

The number of training iterations used was determined by training the models on similar stimuli and noting when the 
networks were able to correctly match at least $90 \%$ all of the training stimuli. This is a typical benchmark of performance as noted in Fallman (1989).

After initial training, each network was tested by training the model using the test stimuli. During this final training period, all three networks used an identical associative learning algorithm (no neuronal death component) to simulate the effects of experiential learning after the critical or sensitive period has passed. The above procedure was repeated ten times for each combination of independent variables. Each time the procedure was repeated, the random noise feature created new distortions of the prototypes for the initial training sequences.

The number of training cycles required for each of the networks to correctly match the test stimuli at the $90 \%$ level was noted. Tables of mean-squared error (calculated by subtracting network output on each cycle from the input pattern, squaring the result and summing for all input elements) were prepared. 
Analysis

A multivariate analysis of variance (MANOVA) was performed on the number of learning cycles required for each model to learn to correctly classify the test stimuli in each of the environmental conditions, and the sum-squared error measured at the start of the test session for each combination of the independent variables. An alpha of .05 was used to asses the results of the analysis.

A MANOVA was specifically selected to allow analysis of the effects of multiple independent variables on 2 correlated dependent variables, while maintaining adequate protection against Type I error (Tabachnick and Fidell, 1983). In addition, the MANOVA allowed examination of the predicted interaction between the 2 independent variables, predicted to occur when an overconnected model is shaped through neuronal death and experiential stabilization in an environment which provides for exposure to certain critical experiences.

Hypothesis. It has been observed that exposure to certain types of stimuli early in life can have a significant impact on the ability to learn related stimuli later in life. Specifically, for certain types of stimuli, early exposure can greatly facilitate later learning. Conversely, lack of early exposure to these same types of stimuli can greatly impair later learning.

It was hypothesized that there is a certain set of physiological characteristics which, when present, will allow 
a neural system to display the observed critical or sensitive period phenomena. These conditions include both an initially overconnected neural structure, and an experientiallymodulated neuronal death component, operating early in the developmental sequence.

This combination of characteristics was hypothesized to produce a form of structural "priming," where the neural structure is modified and stabilized through early experience so as to exhibit differential learning effects to certain types of stimuli when encountered later in development. As neural system characteristics deviate from those hypothesized above, it is less likely that this "priming," and the resultant differential learning effect, will be observed.

It should be noted that the proposed combination of variables may not result in an optimum learning paradigm. There may be other paradigms or sets of conditions which result in more efficient learning mechanisms for a given set of stimuli. The hypothesized combination of variables were expected, however, to provide an efficient means of allowing a given neural structure to efficiently adapt to a wide variety of environments. Most importantly, this combination of variables was expected to produce results that were most closely aligned with those actually observed in developmental studies (i.e., critical or sensitive period phenomena).

Predictions. It was predicted that the ability of the model used in this experiment to learn would not be equal for all combinations of the independent variables. Specific 
predictions about the outcome of the above experiment are detailed below. These outcomes are illustrated in Figure 4 using the dependent variable which measures the number of learning cycles required for the model to correctly learn a given set of test stimuli. The behavior of the 2 dependent variables used in the experiment (number of required learning cycles and sum-squared error) was expected to be highly correlated. Therefore, the results for sum-squared error were expected to be similar.

Prediction 1. A interaction was predicted between neuronal death and experiential sequence. Specifically, it was predicted that there would be fewer training cycles required when neural death is combined with an experiential sequence that provides the variant stimuli in the first training phase. For all other experiential sequences, the model should require fewer training cycles if the neuronal death component is absent.

This interaction, in effect, defines the critical period effect. This combination of independent variables represented what the experimenter believed to represent the physiological and experiential factors necessary to produce a critical or sensitive period effect. Figure 4.a illustrates the predicted interaction.

Insert Figure 4 about here... 
Prediction 2. A main effect was predicted for model type. Specifically, both the genetic and overconnected models were expected to require less training and exhibit lower sumsquared errors than either the experiential or randomized model. This was predicted because the connection weight strengths necessary for the model to respond to the prototype stimuli were initially built in to the genetic and overconnected models but were required to be developed through training in the experiential and random models. Since the models were initially trained for only about one third of the number of training cycles necessary to learn to correctly classify similar stimuli, neither the experiential nor random models should have had adequate time to construct equivalent connections. Figure 4.b illustrates the predicted results associated with model type.

Prediction 3. A main effect was predicted for neuronal death. The neuronal death algorithm results in an effective decrease in learning rate. Learning rate governs the amount of weight change on each learning cycle. Therefore, as learning rate decreases, the number of training cycles required to learn equivalent stimuli should increase. Since neuronal death decreases effective learning rate, the models should have learned more rapidly when the neural death algorithm is not present. Figure 4.c illustrates the predicted results associated with neuronal death.

Prediction 4. A main effect was predicted for experiential sequence. The Hebbian-like learning paradigm 
used in the model is empirical in nature. The more exposure the model has to a given set of patterns, the better it will learn those patterns. Therefore, there should be an inverse correlation between amount of exposure to the prototype variants and the number of cycles required for the model to satisfactorily learn a given set of patterns.

The amount of exposure the model receives for the test stimuli varied across the sequence variable. Sequence 4 (model exposed to the variant patterns on each training cycle) should have resulted in the lowest number of training cycles required to learn the prototype patterns. Sequence 1 (model never exposed to variant stimuli) should have resulted in the most training cycles. Predicted results for experiential sequence are illustrated in Figure 4.d. 


\section{RESULTS}

\section{Summary}

Table 1 summarizes the means and standard deviations of the dependent variables, CYCLES and SSE for all combinations of the independent variables, MODEL, EX_SEQ, and DEATH. When the data were examined across model type (MODEL), it was found that the overconnected model was the most efficient overall in learning the stimulus set as measured by both dependent variables. The overconnected model required the fewest average number of cycles over all trials to learn to correctly identify the prototype stimulus patterns (CYCLES), and exhibited the least sum-squared error (SSE), indicating a close match of the overconnected model's connectivity structure to the prototype patterns. The experiential model was only slightly less efficient overall than the overconnected model for both dependent variables.

\section{Insert Table 1 about here...}

Surprisingly, the genetic model proved least efficient overall when examined using CYCLES data, requiring more training cycles than even the random model. When examined for structural fit, the genetic model still fared poorly, besting only the random model. It is believed that the poor overall performance of the genetic model was due to the large effect of noise-induced structural changes in the genetic model when 
the neuronal death component was present. These effects are discussed in detail below.

The results also indicate differences in both dependent variables of the environmental sequence (EX_SEQ).

Predictably, when the models were not exposed to the variant stimuli during training, both the number of training cycles required to learn the prototype stimuli (CYCLES), and the sum-squared error (SSE) were highest.

It is interesting to note the difference in the models' behavior when the variant stimuli are presented in the first and second half of the training sequence. For both sequences, the actual amount of exposure to the variant stimuli was the same. When the models were exposed to the variant stimuli early in the training phase (First-half), however, the models tended to learn the prototype patterns much more rapidly than if the same amount of stimulus exposure was provided during the second half of the training phase (Second-half). In fact, as indicated in Table 1, presenting the variant stimuli for the first half of the training phase enhanced the model's ability to learn the prototype stimuli (that is, reduced the time to learn as measured by CYCLES) almost as much as presenting the variant stimuli continuously throughout the training phase (All). Continuous presentation of the variant stimuli throughout the training phase, however, did result in the smallest measured sum-squared error (SSE).

Examination of Table 1 also indicates that much more learning was required by the models (as measured by CYCLES) 
when the neuronal death component was present during training. There was a much less pronounced effect for sumsquared error (SSE). Of particular interest is the large increase in the standard deviation for CYCLES when the neuronal death component was present. No such phenomenon was noted for SSE. In fact, the standard deviation for SSE actually decreased slightly when the neuronal death component was present.

\section{ANALYSIS}

A $4 \times 2 \times 4$ between-subjects multivariate analysis of variance was performed on the 2 dependent variables: CYCLES (Number of Required Learning Cycles), and SSE (Sum-Squared Error). Independent variables were MODEL (Model Type), DEATH (Neuronal Death), and EX_SEQ (Experiential Sequence). SPSS-PC MANOVA was used for the analysis. There was a single combination of independent variables (Genetic Model, Deprived Environment, Neuronal Death = True) where the model was unable to successfully classify the stimulus set. Adequate data was obtained for SSE, but the model reached the maximum number of planned training cycles before successfully classifying the stimulus set. Data for CYCLES was entered for this cell as the maximum number of cycles planned for the experiment (150) and included in the analysis. Table 2 summarizes the results obtained in the experiment.

Insert Table 2 about here... 
Using Wilks' criterion, there was a significant multivariate effect $\mathrm{F}(2,287)=5620.27, \mathrm{p}<.001$. In addition, there was a significant interaction effect noted for MODEL by DEATH by EX_SEQ $F(18,574)=1.70, \mathrm{p}<.036$. A significant interaction was also noted for EX_SEQ by DEATH, $F(6,574)=9.89, p<.001$, MODEL by $\operatorname{DEATH}, F(6,574)=3.50, p$ $<.002$, and MODEL by EX_SEQ, $F(18,574)=5.19, \mathrm{p}<.001$. Significant main effects were noted for all 3 independent variables, MODEL, $F(6,574)=59.18, \mathrm{p}<.001$, EX_SEQ, $\mathrm{F}(6,574)=82.88, \mathrm{p}<.001$, and $\operatorname{DEATH}, \mathrm{F}(2,287)=48.08, \mathrm{p}<$ .001

Table 3 summarizes the adjusted sums of Squares and the strength of association $\left(\mathrm{n}^{2}\right)$ for both dependent variables from each of the sources of variance. The strength of association reflects the ratio of the adjusted sum of squares (hypothesis sum of squares times the associated degrees of freedom) for each effect over the total sum of squares as described in Tabachnick and Fidell (1983, page 244). These results reflect moderate association between EX_SEQ and SSE $\left(\mathrm{n}^{2}=.282\right)$, and MODEL and $\operatorname{SSE}\left(\mathrm{n}^{2}=.187\right)$. A less substantial association was attributed to the interaction effect of EX_SEQ BY MODEL on SSE $\left(n^{2}=.117\right)$. There were only minor associations noted between any of the independent variables and CYCLES. These included EX_SEQ $\left(\mathrm{n}^{2}=.054\right)$, DEATH $\left(\mathrm{n}^{2}=.024\right)$, and the interaction of EX_SEQ BY DEATH $\left(\mathrm{n}^{2}\right.$ $=.027)$. 


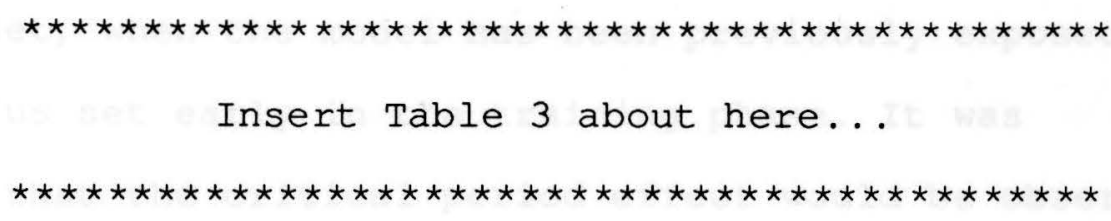

Number of Required Learning cycles

The univariate F-tests that would have resulted if the dependent variables had been assessed separately are summarized in Table 4. There were significant effects on CYCLES for the interaction of EX_SEQ BY DEATH $F(3,288)=$ 1.70, $\mathrm{p}<.001$, and main effects for $\operatorname{DEATH} F(1,288)=70.59$, $p<.001$, and EX_SEQ, $F(3,288)=17.70, p<.001$.

Insert Table 4 about here...

\section{Sum-Squared Error}

The univariate analysis on SSE shows a significant interaction for MODEL BY DEATH BY EX_SEQ F (9,288) = 2.91, p < .003 , the interaction of EX_SEQ BY DEATH, $F(3,288)=8.51, \mathrm{p}$ $<.001$, the interaction of MODEL BY DEATH, $F(3,288)=6.25$, $\mathrm{p}$ $<.001$, and the interaction of MODEL BY EX_SEQ, $F(9,288)=$ 10.52, $\mathrm{p}<.001$. In addition, main effects were noted for

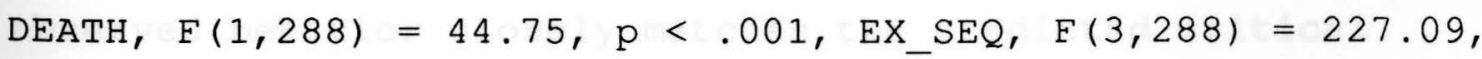
$\mathrm{p}<.001$, and MODEL, $\mathrm{F}(3,288)=150.68, \mathrm{p}<.001$.

\section{Critical Period Effect}

In this experiment, a critical period effect was defined as a significant decrease in the number of learning cycles required for the model to learn to correctly identify a 
stimulus set, when the model has been previously exposed to the stimulus set early in the training phase. It was predicted that the critical period effect would be observed as an interaction effect: learning would be most efficient when model type was an overconnected network (MODEL $=2$ ), the experientially-modulated neuronal component was present $($ DEATH $=$ True), and the environmental sequence resulted in the model being exposed to the prototype stimuli early in the training phase (EX_SEQ $=2$ ). Figure 4 illustrates the predicted pattern of results for the number of required learning cycles.

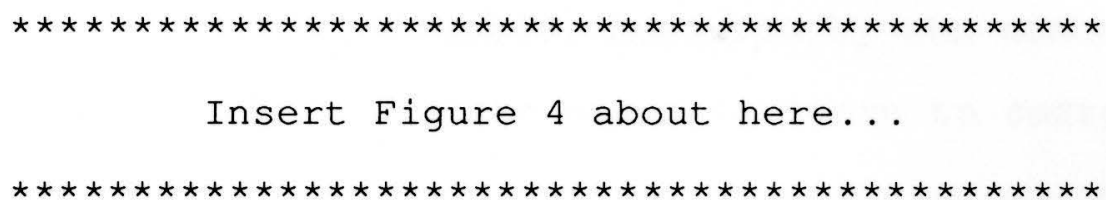

There was no significant interaction effect noted for CYCLES when all 3 independent variables were assessed simultaneously, $F(9,288)=0.39, p>.01$. As discussed earlier, however, there was a significant EX_SEQ BY DEATH interaction effect noted for CYCLES. Figure 5 illustrates the nature of this interaction. It can be seen that the observed behavior closely matches the predicted critical period behavior. That is, there are significantly fewer learning cycles required to correctly learn a given stimulus set when experientially-modulated neuronal death is combined with an environmental sequence where the model is exposed to the stimulus set early in the learning sequence. Although it had been expected that this effect would be restricted to the 
overconnected model, MODEL was not a critical factor in generating the critical period effect.

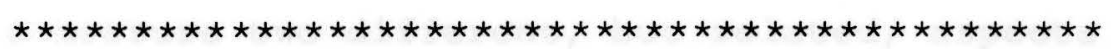

Insert Figure 5 about here...

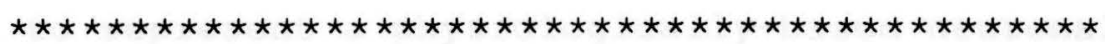




\section{DISCUSSION}

The two dependent variables used in this experiment were designed to measure two different, but related, aspects of the computer-based, associative memory model used in this experiment to represent a physiologically-based neuronal structure. The first aspect, measured by the sum-squared error term is a measure of the goodness of fit of the model's basic "neural structure", that is, the connection weight matrix values. This is an indicator of how well the model has been adapted to the stimulus set by the previous training using noisy input data.

The second dependent variable, measured by the number of learning cycles required for the model to learn to correctly identify the prototype stimulus set after training on noisy data, measures the ability of the model to learn. That is, CYCLES is a measure of both the model's existing structure, and the overall plasticity (effective learning rate) of the available neural connections.

It is possible with the model used in the experiment to have a very well adapted model that exhibits poor overall learning due to the decrease in plasticity level effected by experiential pruning. On the other hand, it is possible to have a model enter the testing phase with a structure poorly adapted to the prototype data set, but with a high level of plasticity. This model will tend to exhibit satisfactory overall learning because the plasticity level is high enough to allow the model to rapidly change its overall connectivity 
pattern ("neural structure"). Thus, it is important to closely examine both dependent variables across all settings.

\section{Critical Period Effect}

It was hypothesized that the combination of an overconnected model, an experientially-modulated neuronal death component which acted to quickly prune out unused neural pathways, and an environmental sequence which exposed the model to variations of the prototype stimuli early in development would interact to produce a significant decrease in the number of learning cycles needed to correctly identify a given stimulus set later in the developmental sequence (i.e., demonstrate a critical or sensitive period effect). This hypothesis proved incorrect in one important aspect. There was, indeed, a significant interaction noted for environmental sequence and the neuronal death component. When the neuronal death component was present, a significant interaction was noted that closely resembled the hypothesized critical period effect. That is, there was a significant decrease in both dependent variables when all of the models were exposed to the prototype variants early in the environmental sequence. Model type, however, was not a factor in producing the expected critical period effect.

\section{Model Type}

Although there was no significant univariate effect of MODEL on CYCLES, it is interesting to examine the behavior of all of the various model types for evidence of the critical period effect. Figure 6 illustrates the results of CYCLES 
when examined across model type. As can be seen, all model types exhibit a behavior that is closely related to the predicted critical period effect. That is, all models exhibit a decrease in the number of cycles required for the model to learn a stimulus set when the set is presented early in the environmental sequence, and an experientially-modulated neuronal death component is present.

Insert Figure 6 about here...

Thus, all model types, when combined with the neuronal death component and the early exposure to the prototype variants, were equally effective in producing a critical period effect. From the results of this experiment, there would appear to be no great advantage conferred on learning by a genetically-determined neural structure. It appears that all that is required to successfully adapt to a given environment is a sufficiently plastic structure, and sufficient early exposure to characteristic stimuli.

There was, however, a significant difference noted among models with regard to sum-squared error. That is, the overconnected model exhibited lower sum-squared error than any other model type. Thus, the overconnected model would appear to adapt more accurately to a given experiential environment, at least at the structural level. However, the overall decrease in plasticity generated by the neuronal death component prevents this structural advantage from being 
expressed in a functional behavior, that is, a reduction in the number of training cycles required to successfully learn the stimulus set.

The findings regarding the neural structure are similar to the results obtained by Spinelli and Jensen (1978). They found that the effects of early experiences were reflected in a straightforward manner in the cortical structures of kittens (i.e., an increase in the number of neurons associated with a given stimulus, and an increase of 3 to 6 times in the diameter of locus of effect).

In their experiment, Spinelli and Jensen found that the changes in the cortical structures were accompanied by demonstrable learning effects. This was not, however, the case in the current experiment where structural change did not always reflect a change in ability to learn. It would appear from Spinelli and Jensen's work that, in real organisms, structural changes may be intimately linked to behavioral changes. That is, although the 2 variables exhibited substantially different behaviors in the model, they may be more closely correlated in actual organisms.

An interesting aspect of the sum-squared error analysis shows that the genetic model exhibited greater sum-squared error (SSE) than either the experiential or the overconnected models. Figure 7 illustrates the observed relationships. The sum-squared error variable is designed to assess the fit of the overall model structure to a given set of stimuli. That is, it is a measure of how well the training stimuli have 
pruned the neural structure to match the prototype stimulus set. It would seem likely that the genetic model would more closely correspond to the prototype structure, even after significant training on the variant stimulus sets. This, however, was not the case. The genetic model consistently exhibited a poorer fit than either the overconnected or experiential models. Only the random model performed worse.

Insert Figure 7 about here...

The poor fit of the genetic model's structure tends to indicate that the genetic model is less efficient at reflecting the effects of environmental stimuli than either the experiential or overconnected paradigms, at least when dealing with noisy environments (remember that a random noise signal was added to the prototype patterns during the initial training sessions).

Collyer, in a series of papers $(1985,1986,1988)$, has examined the goodness of fit characteristics of strong and weak models in cross-validation procedures using clean and noisy data. A strong fitted model is defined as a model with few parameters that can account for a significant portion of the variance in a given data set. A weak fitted model is one that can account for a large amount of variance on a specific set of source data, but at the cost of more free parameters.

In conventional model-fitting situations, a weak fitted model will tend to conform more closely to the data than a 
strong-fitted model. When cross-validated with new data, however, the weak model will tend to fit less closely and the strong model will actually fit best under many conditions. It would appear that the behavior of the genetic model in this experiment corresponds closely to Collyer's analog of a weak model.

To investigate this behavior further, a single set of data runs was made without any noise added to the variant stimulus set. Figure 8 shows the results of these runs. Surprisingly, the genetic model still exhibited poorer performance than either the overconnected or experiential models. Therefore, the observed results are probably due to a more fundamental mechanism, rather than simply the typical behavior of a weak model in cross-validation paradigm.

Insert Figure 8 about here...

Figure 9 provides a closer look at the genetic model's behavior under the no-noise condition. Examination of the figure shows that the genetic model exhibits a clear cut critical period effect for both structure (SSE), and behavior (CYCLES). There is, however, a significant difference between the 2 dependent variables' behavior. CYCLES shows a significant difference between the model's behavior with and without the neuronal death component. SSE, however, exhibits no such difference. The model's resultant structure is unaffected by manipulation of the neuronal death factor. 
Insert Figure 9 about here...

Nature does, in fact, provide for rapid neuronal death. It is unlikely that this mechanism would have evolved without good reason. A further examination of the principles involved in the associative memory model used in the experiment is helpful to clarify these observations.

The variant data sets used for training are actually made up of subsets of the prototype patterns. When an associative memory model is exposed to closely related data (i.e., closely-related subsets), several apparent stages of learning have been observed (Rumelhart and McClelland, 1985). Initially, the models tend to try and learn each individual pattern in the data set. After significant exposure to the overall data set, however, these models tend to extract the central tendency, or mean of the set.

The genetic model is already optimally "tuned" to the prototype data set. When it is first exposed to the variants early in the training phase, it has a high degree of plasticity. It would appear that the genetic model rapidly changes the built-in optimal structure to try to match the actual variant patterns. Rapid deterioration of the built-in structure occurs with the observed loss of learning efficiency and structural fit.

The overconnected and experiential models do not have any specific structure built in to them at the start of training. 
Therefore, when they are exposed to the variant data, they rapidly tend to extract the underlying regularities in the data set. These models appear to rapidly converge on the prototype or underlying "concept" associated with the data. The world into which an animal is born is, in general, a "noisy" environment. Although there is a tremendous amount of similarity in the basic environments in which different species will be born, it is impossible to predict exactly how that environment will be structured, or what detailed experiential history an organism will encounter. The results of this experiment indicate that a prewired genetic structure is not necessarily the most efficient structure for adapting to a noisy environment, and may actually be disadvantageous. In addition, the experiment has shown that the neuronal death component has no effect on the genetic model's behavior.

The lack of effect of the neuronal death component on the genetic model's behavior, coupled with the lower efficiency of the genetic model when trained with noisy data provide evidence to suggest that the genetic model does not represent the best design for intelligent, adaptive organisms.

\section{Environmental Sequence}

Environmental sequence proved to be a significant factor across the experiment. As predicted, all models showed a linear decrease in both dependent variables, as a function of the amount of exposure to the prototype variants. Figure 10 illustrates the relationship of both dependent variables to the amount of stimulus exposure. 
Insert Figure 10 about here...

Because of the empirical nature of the computer-based, associative memory model used in the experiment, it was expected that efficiency of learning would be proportional to amount of exposure to the stimulus. The observed results confirm the predicted relationship between amount of exposure to the stimuli and ability to correctly learn to classify the stimuli. The ability of the model to correctly classify a given stimulus set is directly proportional to the amount of the model's exposure to that stimulus set.

Sequences 2 and 3 both provide the model with the same amount of exposure to the stimuli. The sequence of this exposure, however, is very different. Sequence 2 provides the exposure in the first half of the training phase. Sequence 3, however, provides the exposure in the second training phase. Therefore, examination of the model's behavior for these particular sequences can be particularly enlightening regarding the effects of the timing of experience on the models' behavior.

When the models were exposed to the stimulus set early in training, the ability to learn (CYCLES) was significantly enhanced relative to exposure in the second training phase. The structure of the models (SSE), however, appeared relatively unaffected by the sequence of presentation. Thus, 
structural change seemed to be only a function of the total amount of experience.

This result seems to indicate that, at least for some kinds of experiential deprivation, intervention, in the form of providing an enriched environment later in life, might prove successful. All that is required to adapt the structure is the necessary amount of exposure. A closer look at the data, however, shows that this may not be the case.

Examination of the low-noise data set shows no difference in either dependent variable when the variants were presented in the first training phase or when they were presented for all training cycles. Neither was there any difference when the variants were either not presented at all, or when they were not presented until the second training phase. Obviously, the timing of experiences has a great impact, both on the neural structure, and on the resultant behaviors.

When the models' behavior is examined in regard to the presence or absence of the neuronal death component, the effect is even more pronounced. When the neuronal death component is present, even with noisy data, the models exhibit a definite critical period effect for both CYCLES and SSE. That is, both the structure and the models' overall ability to learn are significantly affected by the order of experiences. Thus intervention may require more than simply providing an enriched environment to overcome early deprivation effects. 
There is still, however, hope for intervention techniques. In all but 1 trial, all models successfully learned the stimulus set. It often required a significantly greater amount of exposure, but eventually, the lack of plasticity was overcome simply by amount of exposure. Therefore, it may be that intensive, prolonged stimulation may overcome deprivation effects. A similar effect has been noted in some cases of brain damage (e.g., stroke) in adults where the level of neural plasticity is assumed to be significantly decreased. Intensive, prolonged rehabilitation has often resulted in at least partial recovery (or relearning) of behaviors and skills that were affected by the injury.

\section{Neuronal Death Effect}

The neuronal death component used in this experiment represents changes in neural plasticity by reducing the overall learning rate applied to a specific group of neurons. Since the ability of the model to learn is proportional to the amount of exposure to the stimulus times the effective learning rate, and the neuronal death component works to reduce the overall learning rate, it is expected that the model should consistently take longer to learn a given stimulus set when the neuronal death component is present. The observed data tend to validate this hypothesis. In all cases, the model requires more learning cycles when the neuronal death component is present, than when it is not. 
Thus it would appear that neuronal death results in a less efficient overall learning mechanism.

The neuronal death component also, however, had a large impact on the variability of CYCLES, the measure of time it took the model to learn. The variability sharply increased when the neuronal death component was present. There was no corresponding effect noted for the structural fit of the model to the prototype patterns (SSE). Thus, it would appear that neuronal death, even though having a profound affect on neural structure, does not, in itself, directly prevent the neural structure from adapting to a given experiential environment. Rather, it would appear that neuronal death acts to modulate the overall plasticity of the neural structures. Rapid neuronal death, at least in the mammalian nervous system, is a well-established fact. Therefore, this death mechanism must convey some overriding survival advantage to an adaptive organism, or it would not have evolved or been quickly weeded out in favor of more efficient mechanisms.

The associative memory model used in this experiment was designed to simulate the functioning of the higher level associative areas of the human cortical structures. The associative areas are evolutionary quite young. It is possible that there has not been enough time to evolve detailed genetic controls for these structures.

A closely-fitting but "not-quite right" structure with a low plasticity may never be able to change enough to allow the structure to completely adapt to a particular 
environment. As plasticity is decreased, the ability to modify the neural structures further is decreased, therefore, the organism is unable to adapt. On the other hand, a poorlyfitted but plastic structure can quickly adapt to any environment, and thus easily overcome the initial deficit due to lack of fit.

In the computer-based associative memory model used in this experiment, the design of the neuronal death algorithm ensures that the learning rate (that is, plasticity) of the neuron circuits which are stimulated by the environment is maintained at a fairly high value. Only circuits which do not actively participate in processing environmental stimuli have their effective learning rate (e.g., plasticity) decreased. The neuronal death algorithm tends to tune not only the actual structure of the model's simulated neuronal circuits themselves, but the overall response characteristics (i.e., plasticity) of those circuits as well.

From a pure learning standpoint, the most efficient model nature could develop would be one with a geneticallydetermined structure that closely matches the environmental stimuli, and a high level of plasticity. This may not, however, be the most efficient overall paradigm in the natural world. Human development includes a relatively long period of helplessness following birth where the neural structure is constantly exposed to representative environmental stimuli. During this period, it would be a simple matter to use experientially-modulated neuronal death 
as a pruning mechanism to adapt the neural structures to reflect the stimuli provided by the existing environment. Given the complexity of developing genetically-controlled structures to cope with all the possible interactions with the environment, the physiological overhead necessary to evolve highly-detailed cortical structures for the higher level associative areas may not even be justified from an evolutionary standpoint.

The results of this experiment, coupled with empirical data regarding neural development, indicate that experientially-modulated neuronal death, acting on an overconnected but non-specific neural structure, provides the most efficient mechanism for allowing an intelligent organism to rapidly adapt to a specific experiential environment. 


\section{SUMMARY, CONCLUSIONS AND IMPLICATIONS}

There is a long history of argument and discussion about the relative import of genetic and experiential components on human development. The current consensus is that genetics and environment contribute equally to the developing organism. The results of this experiment tend to dispute that consensus, however, and lend credence to the experiential, or nurture, side of the argument. It would appear that specific genetic programming (at least at the level of the detailed neural structures) is of little use to an organism developing in a noisy or variable environment.

There is a wealth of evidence available that points to a strong genetic component in the structure of the nervous system. These genetically-controlled structures seem to be associated however with regions of the brain that are less plastic in nature and less well associated with higher level learning phenomena. In the cerebral cortex, functional structure appears to be only roughly defined by genetics and strongly impacted by the result of experience.

Squire (1987) has pointed out that different areas of the brain may apply different types of learning mechanisms. As an example, calpain-fodrin changes the efficiency of synaptic transmission. This change is considered a basic learning mechanism. The calpain-fodrin reaction occurs only in the more recently-developed areas of the brain (cerebral cortex). However, learning has also been demonstrated to take place in the cerebellum which lacks calpain. Therefore, there are 
probably at least 2 forms of learning at work in the brain, and possibly many more.

This experiment was designed to investigate the associative types of learning that are usually associated with the higher cortical structures. It may be that there are critical period phenomena that are based on lower level brain structures that do, in fact, have a strong genetic component. This does not seem to be true for associational learning.

It would appear from the results of this experiment that the nature, amount, and timing of early experience is the most critical factor affecting later learning in the higher cortical regions. The genetic component can vary significantly with little or no impact on later ability to learn. There is some empirical evidence that this, in fact, true for humans. The majority of children labeled mildly retarded exhibit no identifiable brain pathology. Thus, there is no observable reason for the retardation. Most, however, have been found to come from family backgrounds characterized by a lack of intellectual stimulation, and general environmental deprivation (Coleman, Butcher and Carson, 1980)

In mammals, it would appear that an initial overabundance of neural pathways, roughly organized in a generic columnar structure, and rapidly pruned by experientially-modulated death has replaced genetic tuning as the agent responsible for driving behavioral adaptation to a given environment. 
There may be, however, indirect evidence for a genetically-modulated experiential process in the higher level brain regions. There is at least one physiological factor which can have a long term effect on the efficacy of experiential changes in the nervous system. The myelin sheath functions to increase the efficiency of transmission of the action potentials in the neural circuits. If the experiential effect is a function of both the actual stimulus input and the strength of the transmitted impulse, then perhaps the physiological contribution of myelinization is a key factor affecting the experiential structuring of certain regions in the brain.

It has been found that myelinization of different brain regions proceeds at different rates (Gazzaniga, 1985). The cortex, which is the most plastic of the brains regions, does not become fully myelinized until the third decade of growth. Therefore, there may be a link between the degree of myelinization and the level of plasticity of the neural system. It may be that critical period phenomena can only be observed in areas that are fully myelinized. This would explain the drastic difference in time scales between certain critical period effects, such as imprinting, which occurs in a very short time frame, and language learning in children, which occurs over a much longer time interval.

The myelinization process itself, however, may somehow be modulated through an experiential component. At present, there has been little research on either of these 
possibilities, and it represents a potential area of future research.

There may be other considerations. In this experiment, structural change was noted to be affected by the total amount of exposure to the training stimuli, but not by the sequence of exposure. The ability of the model to learn, however, was significantly impacted by the sequence of exposure. When the model was exposed to the training stimuli early in the training phase, plasticity remained high in the associated connectivity matrix entries, and the model's ability to learn later in development was enhanced. If the model was not exposed to the training stimuli until late in the training phase, decreased plasticity (due to experientially-modulated neuronal death) prevented the structural change brought about by experience from being manifested in later learning ability. If it is found that there are, in fact, structural changes taking place in humans due to early experience that are not being exhibited, there is the possibility of successful intervention by chemically manipulating plasticity levels.

Because they operate through fundamentally different mechanisms, the basic neural structure may be formed through the pruning and stabilization of early experience, but the level of plasticity (e.g., the ability to "tune" the baseline neural circuits) may be inadequate. Therefore, the organism is unable to capitalize on the existing structure, that is, to learn in a given environment. Intervention 
techniques may be developed that allow manipulation of the plasticity level in the higher order brain structures that will allow the expression of the structural adaptation.

There has been some pioneering work in chemically manipulating the plasticity level of neurons (Squire, 1987; Aoki and Siekevitz, 1988; Kalil, 1989). This work is not yet, however, well understood or accepted. If there is a strong genetic component in the overall organization of the cortex, then the effects of early deprivation may be overcome through chemically-increased plasticity. The calpain-fodrin mechanism is a direct means of changing the efficacy of a given synapse, and could possibly be an agent through which to modulate neural plasticity. Squire discusses several other possible agents that could modulate plasticity at the level of the individual neuron.

Much more research is required in this area to support a functional understanding of the factors governing plasticity. Such an understanding could, however, provide the key for the development of effective intervention techniques to deal with certain learning and behavioral disorders (e.g., mild retardation). This experiment has given evidence that computer-based associative memory models, incorporating findings and theories of physiological functioning, can aid researchers in gaining that understanding. 


\section{LIST OF REFERENCES}

Alkon, D.L. (1987). Memory Traces in the Brain. Cambridge: Cambridge University Press.

Alkon, D.L. (1989) . "Memory Storage and Neural Systems." Scientific American, 261, 42-50.

Aoki, C. and Siekevitz, P. (1988). "Plasticity in Brain Development." Scientific American, 259, 56-64.

Bolles, R.C. (1979). Learning Theory, Holt, Rinehart and Winston.

Brown, T.H., Chapman, P.F., Kairiss, E.W. and Keenan, C.L. (1988). "Long-Term Synaptic Potentiation." Science, 242, 724728.

Catania, A.C. (1984). Learning, Englewood Cliffs, NJ: Prentice -Hall, Inc.

Carlson, N.R. (1986). Physiology of Behavior. Boston, MA: Allyn and Bacon, Inc.

Coleman, J.C., Butcher, J.N., and Carson, R.C. ((1980). Abnormal Psychology and Modern Life. Glenview, IL: Scott, Foresman and Company. 
Collyer, C.E. (1985). "Comparing strong and weak models by fitting them to computer-generated data." Perception \& Psychphysics, 38, (5), 476-481.

Collyer, C.E. (1986). "Goodness-of-fit patterns in a computer cross-validation procedure comparing a linear and a threshold model." Behavior Research Methods, Instruments, \& Computers, $18,(6), 618-622$.

Collyer, C.E. (1988). "Parameter Estimates depend both on the source model and the fitted model: An example." Bulletin of the Psychometric Society, 26, (4), 289-292.

Douglas, R.J. and Martin, K.A.C. (1990). "Neocortex." In G.M. Shepherd (Ed.) The Synaptic Organization of the Brain. New York: Oxford University Press.

Fallman, W.E. (1989). Apprentices of Wonder. New York: Bantam Books.

Flavell, J.H. (1985). Cognitive Development. Englewood Cliffs, NJ: Prentice-Hall, Inc.

Gazzaniga, M.S. (1985). The Social Brain. New York: Basic Books, Inc. 
Hinton, G.E. and Anderson, J.A. (1981). Parallel Models of Associative Memory. Hillsdale, NJ: Lawrence Erlbaum Associates.

Kalil, R.E. (1989). "Synapse Formation in the Developing Brain." Scientific American, 261, 76-85.

Knapp, A.G. and Anderson, J.A. (1984). "Theory of Categorization Based on Distributed Memory Storage." Journal of Experimental Psychology: Learning, Memory and Cognition, $10,616-637$.

Lerner, R.M. (1986). Concepts and Theories of Human Development. New York: Random House.

Lichtman, J.W. (1977). "The Reorganization of Synaptic Connections in the Rat Submandibular Ganglion During PostNatal Development." J.Physiol, 273, 155-177.

MacLeod, R.B. (1975). The Persistent Problems of Psychology. Pittsburgh, PA: Duquesne Press.

McClelland, J.L. and Rumelhart, D.E. (1985). "Distributed Memory and the Representation of General and Specific Information." Journal of Experimental Psychology, 114, 159188 
Munro, P.W. (1986). "State-Dependent Factors Influencing Neural Plasticity: A Partial Account of the Critical Period." In J.L. McClelland and D.E. Rumelhart (Eds.) Parallel Distributed Processing. Cambridge, MA: Bradford Books / MIT Press.

Purves, D. and Lichtman, J.W. (1980). "Elimination of Synapses in the Developing Nervous System." Science, 210, 153-157.

Rakic, P. and Riley, K.P. (1983). "Overproduction and Elimination of Retinal Axons in the Fetal Rhesus Monkey." Science, 219, 1441-1444.

Restak, R.M. (1988). The Mind. New York: Bantam Books.

Scott, J.P. (1962). "Critical Periods in Behavioral Development." Science, 138, 949-957.

Schwartz, B. (1989). Psychology of Learning and Behavior. New York: W.W. Norton and Company.

Smoliar, S.W. (1989). "The Challenge of Neural Darwinism." Proceedings of the Eleventh International Joint congress on Artificial Intelligence, San Mateo, CA: Morgan Kaufmann Publishers. 
Stillings, N.A., et. al (1989). Cognitive Science, Cambridge, MA: MIT Press.

Squire, L.R. (1987). Memory and Brain. New York: Oxford University Press.

Spinelli, D.N., and Jensen, F.E. (1978). "Plasticity: The Mirror of Experience." Science, 203, 75-78.

Tabachnick, B.G., and Fidell, L.S. (1983). Using Multivariate Statistics. New York: Harper Row. 
APPENDIX A

MODEL SOURCE CODE LISTING

Program Deathnet;

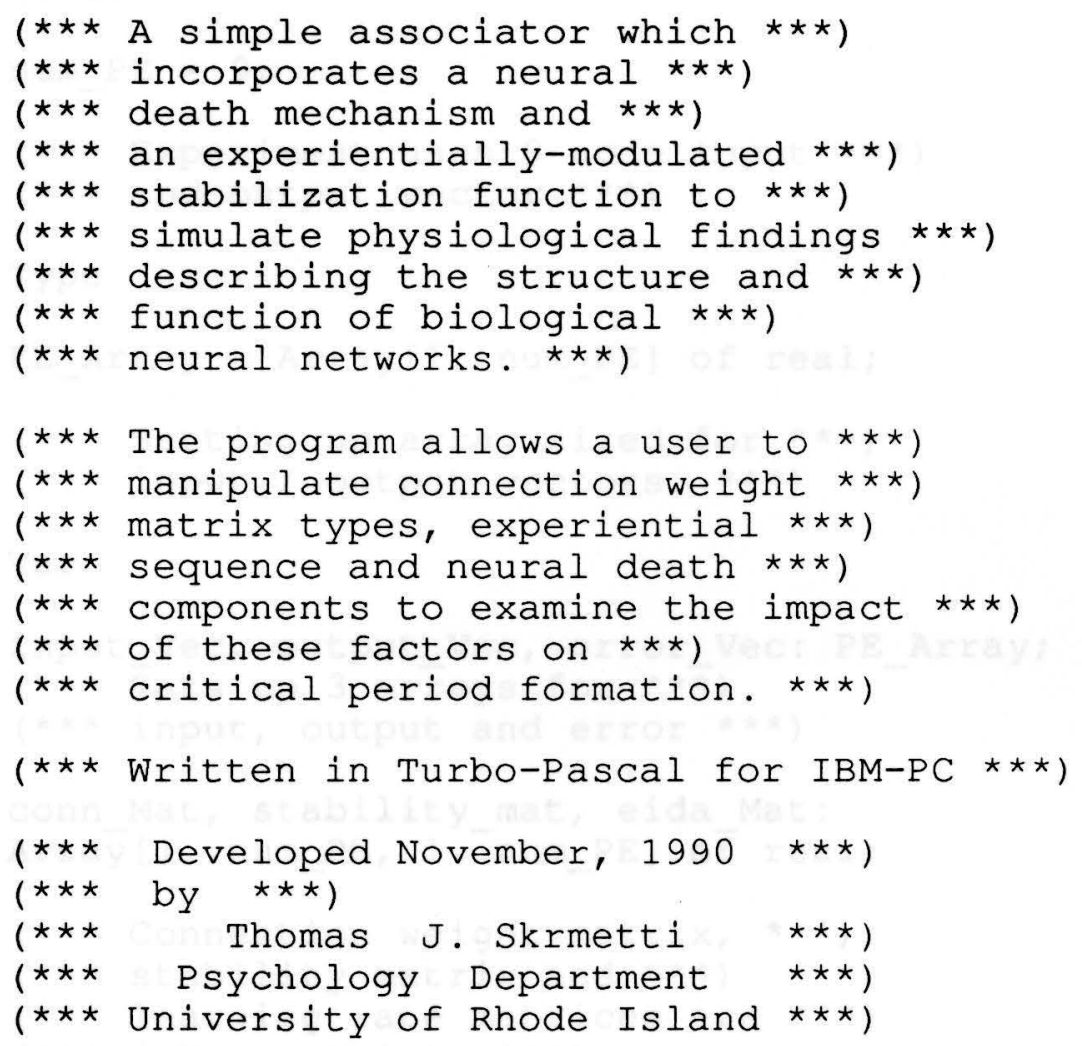




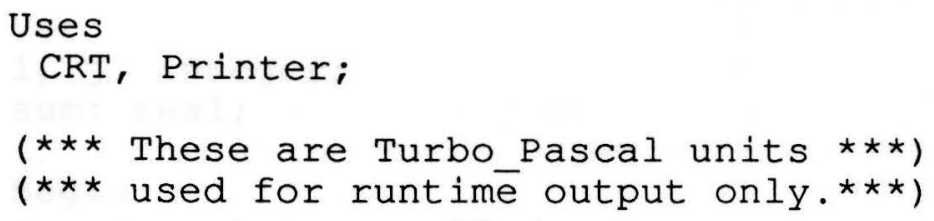

Const

num_PE $=9$;

( $\star \star \star$ Experiment uses 9 node input $\star \star \star)$ ) $(\star \star \star$ and output vector $\star \star \star)$ )

Type

PE_Array $=$ Array $[1 \ldots$ num_PE $]$ of real;

( $\star \star \star$ Setting up array sized for $\star \star \star *)$

( $\star \star \star$ input / output vectors. $* \star \star)$

$\operatorname{Var}$

input_Vec, output_Vec, error_Vec: PE_Array; ( $\star \star \star$ Sets up 3 ar $\bar{r}$ ays for $\star \star \bar{\star}$ )

( $\star \star \star$ input, output and error $\star \star \star$ )

conn_Mat, stability_mat, eida_Mat:

Arraȳ[1..num_PE, 1..num_PE] of real;

( $\star \star \star$ Connection weight matrix, $\star \star \star *)$

( $* \star *$ stability matrix and $* \star *$ )

$(* * *$ learning rate matrices are $* * *)$

$(* \star \star n \mathrm{n} X \mathrm{n}$ matrices $\star \star \star)$

( $* \star \star$ where $\mathrm{n}=$ number of elements $\star \star \star *)$

( $* * *$ in input and output vectors. $* * *$ )

eida, lower_limit, random_weight, choice, sum_squared_error: real $\bar{i}$

count, cycles, iter, entry, Num_Training_Cycles, num_patterns, Model, couñt_i, couñt_j, i, j, code: integer;

filename: text;

dataset, testset, weight_entry, weight matrix, weight, mödel type,

sequence, death_wish, learning_set: string;

input entry: chār;

neurā_death, learning_check: boolean;

Procedure compute_output_vector; 
Var

$i, j$ : integer;

sum: real;

Begin

for $j:=1$ to num PE do

Begin

sum : $=0$;

for $i:=1$ to num $P E$ do

sum := sum +

conn_Mat $[j, i]$ * input_Vec [i] ;

outpūt_Vec[j] := sum;

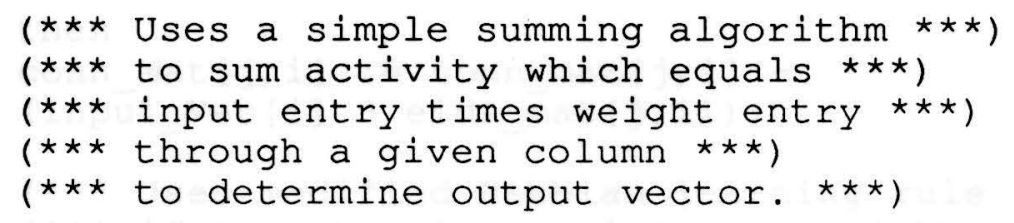

End;

End;

Procedure Update_Conn_Mat_Weights;

Var

$i, j:$ integer;

Begin

If ( (Model $=1)$ and

(learning_set $=$ 'second') )

then Delāy (0) ( $\star \star \star \star$ Skips update $\star \star \star)$ )

else Begin

$(\star \star \star$ The genetic model approximates $\star \star \star)$

$(* \star \star$ the effect of a rapid, $* \star \star)$ )

( $\star \star \star$ genetically-programmed $\star \star \star *)$

$(\star \star \star$ drop in plasticity. $\star \star \star)$

( $* \star \star$ Therefore, the genetic model $\star \star \star *)$

( $\star \star \star$ will not exhibit the ability $\star \star \star *)$

( $* \star \star$ to change connection weight $* \star *$ )

( $* \star \star$ strengths after first training set. $* \star \star)$ )

( $* \star \star$ Program simulates this by skipping $* \star \star$ )

( $* \star \star$ this procedure for second learning $* \star \star$ )

$(\star \star \star$ set of genetic model (Type 1$) . \star \star \star *)$

( $* \star \star$ Model allowed to learn in test set $\star \star \star$ )

( $* \star \star$ only to allow comparison in MANOVA. $* \star \star *)$

( $\star \star \star$ Otherwise data not expected to be $\star \star \star *)$

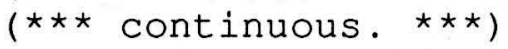

( $\star \star \star$ Model 1 would always "hit limit." $\star \star \star$ )

for $j:=1$ to num_PE do

for $i:=1$ to num_PE do 
Begin

if ( input Vec $[j]=1.0$ )

and (output_Vec $[j]<1.0)$ )

then

conn_Mat $[j, i]:=$ conn_mat $[j, i]+$

(inpūt_Vec[i] * eida_mat $[j, i])$

else if

( (input_Vec[j] = lower_limit) and

(output_Vec[j] > lower_limit))

then

conn_Mat $[j, i]:=$ conn_mat $[j, i]-$

(input_Vec[i] * eida_mat $[j, i])$;

( $\star \star \star$ Uses modified Hebbian learning rule $\star \star \star$ )

( $\star \star \star$ if output < input, increase weight $\star \star \star$ )

( $* \star \star$ if output $>$ input, decrease weights $* \star \star)$ )

( $\star \star \star$ Uses element [j] as the net is $\star \star \star)$ )

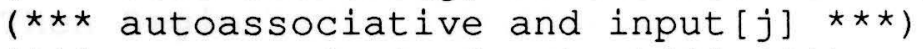

$(\star \star \star$ same as desired output [j] $\star \star \star *)$

if input_Vec[i] * input_Vec[j] $<0$

then stability_mat $[i, j]^{-}:=1.0 ;$

( $* \star *$ If cell affected through experience $* \star \star$ )

$(* \star \star$ note this in stability matrix entry $\star \star \star *)$

( $\star \star \star$ Info used in neural death routine $\star \star \star *$ )

( $\star \star \star$ to limit death of "useful" neurons $\star \star \star *)$

End;

End;

End;

Procedure Initialize_Stability_Matrix;

Begin

for $i:=1$ to num $P E$ do

for $j:=1$ to num_PE do

Begin

stability Mat $[i, j]:=0$;

eida_Mat $[\bar{i}, j]:=$ eida;

( $\star \star \star$ Initially, all cells change $\star \star \star$ )

$(\star \star \star$ with same rate. $\star \star \star)$ )

( $* \star *$ Rates will be modified by neural $* * *$ )

( $* \star \star$ death routines based on $\star \star \star$ ) 
(*** different experiential histories $\star \star \star)$

End;

End;

Procedure init;

Var

$i, j:$ integer;

matrix_entry: real;

Begin

clrscr;

writeln;

writeln;

writeln;

writeln;

writeln;

writeln;

('Initializing connection weight matrix...');

If Model $=1$

Then Begin

weight_matrix := 'Lorenz';

assign (filename, weight_matrix);

reset (filename);

( $* \star \star$ Genetic model connection weights $\star \star \star$ )

( $* \star *$ determined by training $* \star *$ )

( $\star \star \star$ experiential model on prototypes $\star \star \star$ )

( $\star \star \star$ and writing resultant weights $\star \star \star *)$

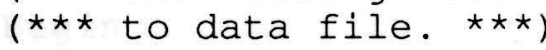

$(* \star \star$ This routine reads in those $\star \star \star *)$

$(\star \star \star$ stored weights $\star \star \star)$ )

writeln;

writeln

('Reading in pre-programmed weights...');

for $i:=1$ to num_PE do

for $j:=1$ to num_PE do

Begin

readln

(filename, weight);

Val

(weight, conn_Mat $[i, j]$, code) ; 
End;

delay (2000);

clrscr;

close (filename);

End;

If Model $=2$

Then for $i:=1$ to num PE do

for $j:=1$ to num PE do

conn_Mat $[i, j]:=\overline{0} .0$;

( $\star \star \star$ Experiential model cells $\star \star \star *)$

$(* \star \star$ start out $0 * \star \star)$

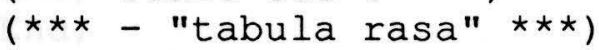

If Model $=3$

Then for $i:=1$ to num $\mathrm{PE}$ do

for $j:=1$ to num PE do

conn_Mat $[i, j]:=\overline{0} .023$;

( $* \star \star * 0.023$ times number of cells (81) $* \star \star *)$

( $\star \star \star *$ equals approximately twice $\star \star \star$ )

$(\star \star \star$ the average entry value $\star \star \star)$ )

( $* * *$ of genetic model connection weights $\star \star \star *)$

( $\star \star \star *$ Factor consistent with findings of $\star \star \star *$ )

$(\star \star \star$ Lichtman and Purves. $\star \star \star)$ )

If Model $=4$

Then

Begin

Randomize;

for $i:=1$ to num $P E$ do

for $j:=1$ to num_PE do

Begin

random weight := random;

if random weight $>0.5$

then Begin

( $\star \star \star$ Determine if entry will be $\star \star \star$ )

( $\star \star \star *$ positive or negative $\star \star \star$ )

$(* \star *>0.5$ positive, < 0.5 negative. $* \star \star)$

random weight := random / 2 ;

conn_Māt $[i, j]:=$ random_weight;

(*ᄎ* Maximum value for any cell $\star \star \star$ ) 


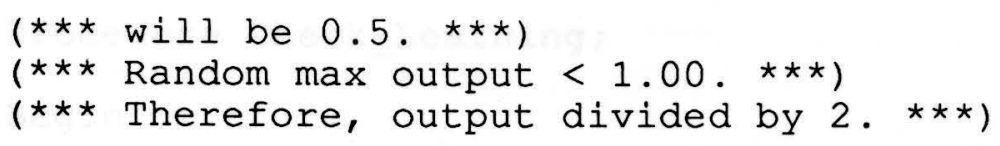

End

else Begin

$(* \star \star<0.5$ will be negative $* * *)$

random weight := random / 2 ;

conn_Māt $[i, j]:=-1$ * random_weight;

End;

End;

End;

delay (2000);

clrscr;

End;

Procedure Sum_Squared_Errors;

Begin

For iter := 1 to num_PE do

Begin

Error_vec[iter] :=input_Vec[iter] - output_Vec[iter];

Sum_Squared_Error := Sum_Squared_Error

$+(\bar{E} r r o r$ Vec $[$ iter] * Errō_Vec [iter]);

( $\star \star \star$ On each learning cycle, $\star \star \star \star *)$

( $\star \star \star$ corresponding elements $\star \star \star *)$

( $\star \star \star$ of input and output vectors $\star \star \star$ )

( $* \star \star$ are compared and error signal $\star \star \star$ )

( $\star \star \star$ computed and summed across all $\star \star \star *)$

( $\star \star \star$ elements and patterns $\star \star \star)$ )

( $\star \star \star$ in each learning cycle. $\star \star \star)$ )

If ( (Sum_Squared_Error $=0.0$ )

and (leārning_set $=$ 'test'))

Then cycles $:=$ Num_Training_Cycles;

( $* \star \star$ If no error, training complete. $\star \star \star *)$

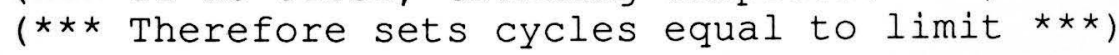

( $* \star \star$ to end "For loop" $\star \star \star *)$

$(* \star *$ Inelegent way to end program... $* \star \star)$

End;

End; 
Procedure Check_Learning;

Begin

For iter $:=1$ to num $P E$ do

If output_vec[iter] $\overline{>}=0.9$

then output_Vec[iter] $:=1.0$

else if output Vec [iter] $<=0.1$

then output_Vec $[$ iter] $:=0.0$;

( $* * *$ Model uses criterion of output $* * *$ )

( $\star \star \star *$ being within $10 \%$ of input. $\star \star \star *)$

( $\star \star \star$ If so, pattern accepted as being $\star \star \star$ )

( $* \star \star$ correctly learned. $* \star \star$ )

( $\star \star \star$ Procedure also acts as a $\star \star \star$ )

$(* \star \star$ limiting function $* \star \star)$ )

( $* \star \star$ to clamp output values between $* \star \star$ )

$(* \star \star$ limits of 0 and $1 . * \star *)$

Learning_Check := True;

For iter := 1 to num_PE do

If input_Vec[iter] $<>$ output_Vec[iter]

then Learning_Check := False;

( $\star \star \star$ Check if output vector $\star \star \star)$ )

( $\star \star \star \star$ equals input vector. $* \star \star *$ )

( $\star \star \star$ That is, has Model correctly $\star \star \star$ )

$(\star \star \star$ learned pattern yet ? $* \star \star)$

If Learning_Check $=$ True

then writeln

('Pattern number ', count, ' learned sat.');

End;

Procedure Kill_neurons;

Var

$i, j$ : integer;

Begin

for $i:=1$ to num $P E$ do

for $j:=1$ to num $P E$ do

if stability_mat $[\bar{i}, j]=0$

then

eida_Mat $[i, j]:=$ eida_Mat $[i, j]$ (eidāMat $[i, j] / 2)$;

$(\star \star \star$ If no cell activity $\star \star \star)$ )

$(\star \star \star$ on learning cycle, $\star \star \star *)$

$(\star \star \star$ reduce eida towards $0 . * \star \star)$ 
( $\star \star \star$ Reflects decrease in $\star \star \star)$ )

$(* \star \star$ effective learning rate $\star \star \star *)$

$(\star \star \star$ due to neuron loss $\star \star \star)$ )

$(* \star \star$ rather than change in plasticity. $* * *$ )

$(\star \star \star$ Note: Most nets use simple global $\star \star \star)$ )

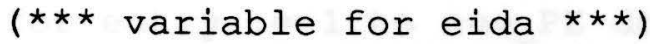

( $* \star \star$ rather than entire separate matrix $\star * \star$ )

( $\star \star \star$ Use of matrix allows simple $\star \star \star$ )

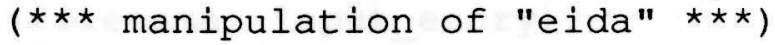

( $\star \star \star$ for selected groups of neurons $\star \star \star *)$

$(\star \star \star$ based on experience. $\star \star \star)$ )

( $\star \star \star$ Algorithm never quite kills all $\star \star \star$ )

( $\star \star \star$ possible paths $\star \star \star)$ )

( $\star \star \star *$ Stabilized neurons less likely $\star * \star$ )

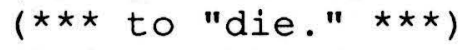

$(* \star \star$ Death rate rapidly decreases $\star \star \star)$ )

( $\star \star \star$ through "time" as measured by $\star \star \star *)$

( $\star \star \star$ number of cycles occuring. $\star \star \star$ )

writeln;

writeln;

End;

Procedure Get_Input_Vector;

Begin

readln (filename);

( $\star \star \star$ Reads over pattern identifier not $* \star \star)$ )

$(\star \star \star$ used in program $\star \star \star *)$

for entry $:=1$ to num $P E$ do

Begin

readln

(filename, input_entry);

if input_entry $=$ ' 1 '

then

input_Vec[entry] := 1.0

else

input Vec [entry] :=

lower-limit;

End;

End;

Procedure Noisy_Input;

Begin

readln (filename); 
( $\star \star \star$ Reads over pattern identifier not $\star \star \star)$ )

$(\star \star \star$ used in program. $\star \star \star)$ )

Randomize;

for entry := 1 to num $P E$ do

Begin

readln

(filename, input entry);

if input entry $=-11$ '

then

input Vec [entry] : = 1.0

else

input_Vec[entry] := lower_limit;

choice := random;

if ( $($ input_Vec [entry] $=1.0)$ and (choice $>0.88)$ )

then

input_Vec[entry] :=0.0

else $\overline{i f}$

$(($ input_Vec[entry] $=$ lower_limit) and (choice $>0.88)$ )

then

input_Vec[entry] := 1.0;

( $\star \star \star$ Reads input file and adds $\star \star \star$ )

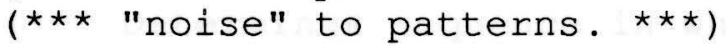

$(* \star \star$ If prob $>0.88$ ( 1 out of 9$), * \star *)$

$(\star \star \star$ change entry. $\star \star \star)$

$(\star \star \star$ If prob $<0.88, \star \star \star)$

$(* \star *$ entry stays the same. $* \star \star)$

End;

End;

Procedure Output_Connection_Weight_Matrix;

Begin

for count_i := 1 to num_PE do

Begin

writeln (lst);

for count_j:= 1 to num_PE do write

(lst, conn Mat [count i, count_j]: $6: 2,{ }^{\prime}$ ') ;

End;

writeln (lst);

writeln (lst); 
End;

Procedure Input_Startup_Values;

Begin

clrscr;

writeln

('(Genetic $=1 ;$ Experiential $=2$;

Overconnected $=3$; Random $=4:$ ) ');

writeln;

writeln ('Enter desired type of model: ');

readln (Model);

writeln

(lst, 'Model type is ', Model, '.');

writeln;

init;

write

('Enter desired experiential sequence: ');

readln (sequence);

writeln (lst,

'Experiential sequence $=$ ', sequence, '. ') ; writeln;

$(* \star *$ Determines sequence in which $\star * \star$ )

( $\star \star \star$ prototypes presented. $\star \star \star *)$

$(\star \star \star 1=$ prototypes never presented $\star \star \star)$

$(\star \star \star 2$ - prototypes presented first. $\star \star \star)$ )

$(\star \star \star 2=$ prototypes presented $\star \star \star)$

( $* \star \star$ training set only. $\star \star \star *)$

$(\star \star \star 3=$ prototypes presented second $\star \star \star)$ )

( $* \star *$ training set only. $* * \star)$

$(\star \star \star 4=$ prototypes presented all $\star \star \star *)$

$(\star \star \star$ training sets $\star \star \star)$

write

('Is neural death a factor ( $\mathrm{Y} / \mathrm{N})$ ? ');

readln (death_wish);

if ( (death wish $=$ ' $Y$ ')

or (death_wish $=$ ' $y$ '))

then neurāl_death := True

else neural_death := False;

writeln

(1st, 'Neural death is ', neural_death, '.');

writeln;

writeln;

write 
('Enter lower limit (-1.0 or 0.0): ');

readln (lower_limit);

writeln;

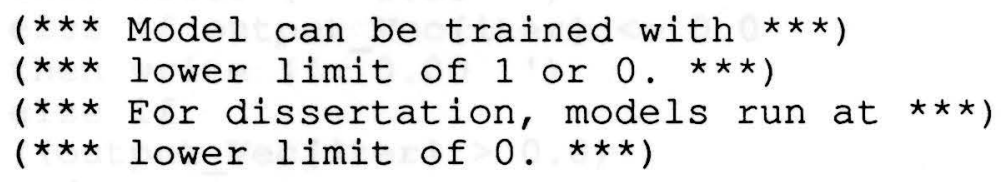

write ('Enter desired learning rate: ');

readln (eida);

writeln

(lst, 'Learning rate $=$ ', eida:6:4,'.');

( $* * *$ Obtains initial value for $* * *$ )

$(\star \star \star$ learning rate parameter. $\star \star \star)$ )

writeln; ('Enter number desired training cycles:');

readln (Num_Training_Cycles);

writeln

(lst, 'Number of training cycles = ', num_training_cycles, '.');

writeln (lst);

Delay (2000);

clrscr;

Randomize;

$(* \star \star$ Sets up seed for $\star \star \star)$ )

( $\star \star \star$ random number generator. $\star \star \star *)$

End;

Procedure Display_Input_Pattern;

Begin

writeln ('Pattern Number: ', count, ':');

writeln;

write (' Input: ');

for iter := 1 to num_PE do

write

(input_Vec [iter]: 6:2, ' ');

End;

Procedure Display_output_Vector;

Begin 
write ('Output: ') ;

for iter $:=1$ to num_PE do

if output_Vec[iter] $>=1.0$

then write (' 1.00 ')

else if output Vec [iter] $<=0.0$

then write ('-0.00 ')

else if

( (output Vec [iter] $>0.0$ )

and

(output Vec $[$ iter $]<1.0$ ))

then write

(output_Vec[iter]: 6: 2, ' ');

writeln;

End;

Procedure Set_Up_First_File;

Begin

learning_set $:=$ 'first';

If $\left(\right.$ sequence $\left.=' 1^{\prime}\right)$ or $\left(\right.$ sequence $\left.={ }^{\prime} 3^{\prime}\right)$ )

then dataset $:=$ 'nopro'

else dataset := 'pro';

assign (filename, dataset);

reset (filename);

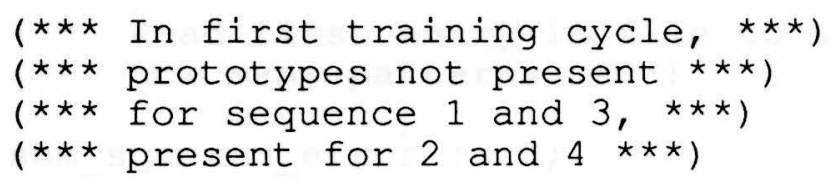

End;

Procedure Set_Up_Second_File;

Begin

learning_set $:=$ 'second';

If $\left(\right.$ (sequence $\left.=\prime^{\prime} 1^{\prime}\right)$ or $\left(\right.$ sequence $\left.=\prime^{\prime}\right)$ )

then dataset $:=$ 'nopro'

else if ( (sequence $\left.=\prime^{\prime} 3^{\prime}\right)$ or $\left(\right.$ sequence $\left.=\prime^{\prime} 4^{\prime}\right)$ )

then dataset $:=$ 'pro';

assign(filename, dataset);

reset (filename);

( $* \star \star$ In second training cycle, $* \star \star *)$

$(\star \star \star$ prototypes not present $\star \star \star)$ )

( $\star \star \star \star$ for sequence 1 and $2, \star \star \star$ )

$(* \star *$ present for 3 and $4 * \star \star)$

End;

Procedure Set_Up_Test_File; 
Begin

learning_set $:=$ 'test';

dataset $\overline{:}=$ 'pro';

assign (filename, dataset);

reset (filename);

( $\star \star \star$ Prototypes are always present $\star \star \star$ )

$(* \star \star$ during testing. $* \star *)$

End;

Procedure Learn;

Begin

For cycles:= 1 to Num_training_cycles do

Begin

writeln;

writeln

('Training cycle number ', cycles,'.');

writeln;

readln (filename, num_patterns);

( $* \star \star$ Read first entry in file to see $\star \star \star$ )

( $\star \star \star$ how many patterns. $\star \star \star$ )

sum_squared_error : $=0$;

( $\star \star \star *$ Initialize error to $0 * \star \star$ )

( $\star \star \star$ before each training cycle. $* \star \star)$ )

For count $:=1$ to num_patterns do

Begin

if learning_set $=$ 'test'

then Get_Input_Vector

else Noisy_Input;

( $* \star \star$ Only add noise on training cycles $\star \star \star$ )

( $\star \star \star$ Testing occurs with $* \star \star$ )

$(\star \star \star$ "clean" prototype...***)

Compute Output Vector;

Update_Conn_MaẼ_Weights;

Display_Input_Pattern;

Display_output_Vector;

Check_Learning; 
$(* \star \star$ See if pattern has been $\star * \star)$

( $* * *$ learned correctly yet... $* * *$ )

Sum_Squared_Errors;

(*ᄎ* Compute current error values $\star \star \star)$

End;

Writeln

(lst, 'Sum Squared Error for cycle ', cycles, ' = ', Sum_Squared_Error:

$6: 4, ' \cdot 1) ;$

If ( (neural death = True)

and (learning_set = 'first'))

then Kill_Neurons;

( $\star \star \star$ Death component only applicable $\star \star \star)$ )

$(\star \star \star$ to first cycle. $\star \star \star)$

reset (filename);

$(* \star \star$ Back to top of input file $\star \star \star)$ )

$(\star \star \star$ for next training cycle $\star \star \star)$ )

End;

close (filename);

( $* \star \star$ close current training file $\star \star \star)$

End;

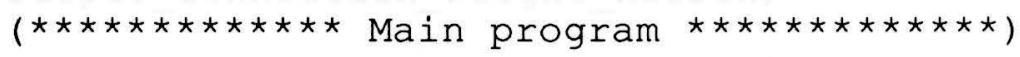

Begin

Input_Startup_Values;

$(\star \star \star$ Set up initial parameters $\star \star \star)$ )

( $\star \star \star$ for each individual run. $\star \star \star *)$

Writeln (lst);

Writeln (lst,

'Initial connection weight matrix: ');

Output_Connection_Weight_Matrix;

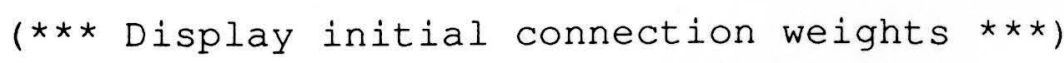

Initialize_Stability_Matrix; 
( $* \star \star$ Sets up initial stability and $\star \star \star)$ )

( $* \star *$ learning rate matrices. $\star * \star$ )

Set_Up_First_File;

Learn;

( $* \star \star$ Read in first pattern set $\star \star \star$ )

( $* \star \star$ and train model. $* \star \star$ )

Set_Up_Second_File;

( $* \star \star$ Change inputs to reflect $\star * \star$ )

( $\star \star \star$ experiential sequences. $\star \star \star \star)$ )

Learn;

Set_Up_Test_File;

num_trāining_cycles :=

num training cycles * 15 ;

Learn;

( $* \star \star$ After both training cycles, $\star \star \star *)$

( $\star \star \star *$ read prototype patterns $\star \star \star$ )

$(* \star *$ Increase number of $\star * \star)$

( $* \star *$ training cycles sufficiently $* \star \star$ )

( $* * *$ to allow all models $* * *$ )

( $\star \star \star$ sufficient number of cycles $\star \star \star *)$

( $* * \star$ to correctly learn patterns. $* * *$ )

writeln (lst);

Writeln (lst,

'Final connection weight matrix: ');

Output_Connection_Weight_Matrix;

$(\star \star \star$ Show final connectivity matrix $\star \star \star)$ )

$(* \star \star$ for each run. $\star \star \star)$ )

End. 
APPENDIX B

DATA SETS

\begin{abstract}
"Pro"
Pattern $1 \quad 100100100$

Pattern 2010010010

Pattern 3001001001
\end{abstract}

"Nopro"

Pattern $1 \quad 011100000$

Pattern $2 \quad 100010111$

Pattern $3 \quad 001100111$ 
APPENDIX C

SUMMARY OF MEANS (CYCLES)

\section{Experiential Sequence}

None 1st 2nd All

$\begin{array}{lllll}\text { No Death } & 20.4 & 15.8 & 20.4 & 14.8\end{array}$

Genetic Model

$\begin{array}{lrrrr}\text { Death } & 60.9 & 25.4 & 46.3 & 25.2 \\ \text { No Death } & 19.7 & 16.4 & 14.7 & 10.5\end{array}$

Experiential Model

$\begin{array}{lrrrr}\text { Death } & 44.8 & 17.1 & 38.4 & 27.5 \\ \text { No Death } & 18.6 & 15.9 & 14.6 & 10.6\end{array}$

Overconnected Model

$\begin{array}{lllll}\text { Death } & 47.1 & 22.8 & 39.1 & 15.5 \\ \text { No Death } & 21.6 & 18.1 & 17.6 & 10.8\end{array}$

Random Model

$\begin{array}{lllll}\text { Death } & 61.1 & 18.8 & 37.9 & 23.6\end{array}$




\section{APPENDIX C \\ SUMMARY OF MEANS (SSE)}

$\begin{array}{ccc} & \text { Experiential Sequence } \\ \text { None } & \text { 1st } & \text { 2nd }\end{array}$

All

No Death

5.97

3.74

5.95

3.67

Genetic Model

$\begin{array}{lllll}\text { Death } & 5.99 & 3.85 & 5.90 & 3.90 \\ \text { No Death } & 5.38 & 3.88 & 3.06 & 1.89\end{array}$

Experiential Model
Death
5.39
4.35
4.22
2.39
No Death
5.23
3.47
2.95
1.57

Overconnected Model
Death
5.14
4.01
4.13
2.15
No Death
7.77
5.26
5.59
2.59

Random Model
Death
7.27
6.52
6.83
5.07 
Table 1. Summary of Means and Standard Deviations

IV

$$
\text { CYCLES }
$$

$\mathrm{m}$
DV

SSE

m $\quad \mathrm{SD}$

\section{MODEL}

$\begin{array}{ccccc}\text { Genetic } & 28.65 & 25.17 & 4.87 & 1.11 \\ \text { Experiential } & 23.64 & 22.43 & 3.82 & 1.26 \\ \text { Overconnected } & 23.02 & 19.88 & 3.58 & 1.27 \\ \text { Random } & 26.19 & 23.60 & 5.86 & 2.04\end{array}$

EX_SEQ

$\begin{array}{ccccc}\text { None } & 36.77 & 27.00 & 6.02 & 1.12 \\ \text { First-half } & 18.79 & 11.71 & 4.38 & 1.20 \\ \text { Second-half } & 28.62 & 21.28 & 4.83 & 1.52 \\ \text { All } & 17.31 & 14.50 & 2.90 & 1.39\end{array}$

\section{DEATH}

$\begin{array}{lllll}\text { No } & 16.28 & 6.99 & 4.25 & 1.77 \\ \text { Yes } & 34.47 & 28.99 & 4.82 & 1.62\end{array}$


Table 2. Summary of MANOVA Results

$\begin{array}{lccccc}\text { Effect } & \text { Value } & \text { App F } & \text { Hyp DF } & \text { Err DF } & \text { Sig F } \\ \text { MxESxD } & .90151 & 1.69684 & 18.00 & 574.00 & 0.360 \\ \text { ESxD } & .82133 & 9.89386 & 6.00 & 574.00 & <.001 \\ \text { MxD } & .93063 & 3.50137 & 6.00 & 574.00 & 0.002 \\ \text { MxES } & .73980 & 5.18613 & 18.00 & 574.00 & <.001 \\ \text { D } & .74903 & 48.08142 & 2.00 & 287.00 & <.001 \\ \text { ES } & .28708 & 82.88447 & 6.00 & 574.00 & <.001 \\ \text { M } & .38171 & 59.17672 & 6.00 & 574.00 & <.001 \\ \text { Const } & .02490 & 5620.26805 & 2.00 & 287.00 & <.001\end{array}$


Table 3. Summary of Adjusted Sum of Squares and $n^{2}$

\begin{tabular}{|c|c|c|c|c|}
\hline & \multicolumn{2}{|c|}{ CYCLES } & \multicolumn{2}{|c|}{ SSE } \\
\hline Source of Variance & SS ${ }^{\prime}$ & $n^{2}$ & SS ${ }^{\prime}$ & $n^{2}$ \\
\hline MODEL & 4,782 & .004 & 792 & .187 \\
\hline DEATH & 26,463 & .024 & 26 & .006 \\
\hline$E X \_S E Q$ & 59,742 & .054 & 1191 & .282 \\
\hline EX_SEQ X DEATH & 29,793 & .027 & 45 & .011 \\
\hline MODEL X DEATH & 1,083 & $<.000$ & 33 & .008 \\
\hline EX_SEQ X MODEL & 10,584 & .009 & 495 & .117 \\
\hline EX_SEQ X MOD X DEATH & 11,808 & .011 & 135 & .032 \\
\hline ERROR & 971,829 & & 1512 & \\
\hline TOTAL & 116,084 & & 4229 & \\
\hline
\end{tabular}


Table 4. Summary of Univariate Analysis

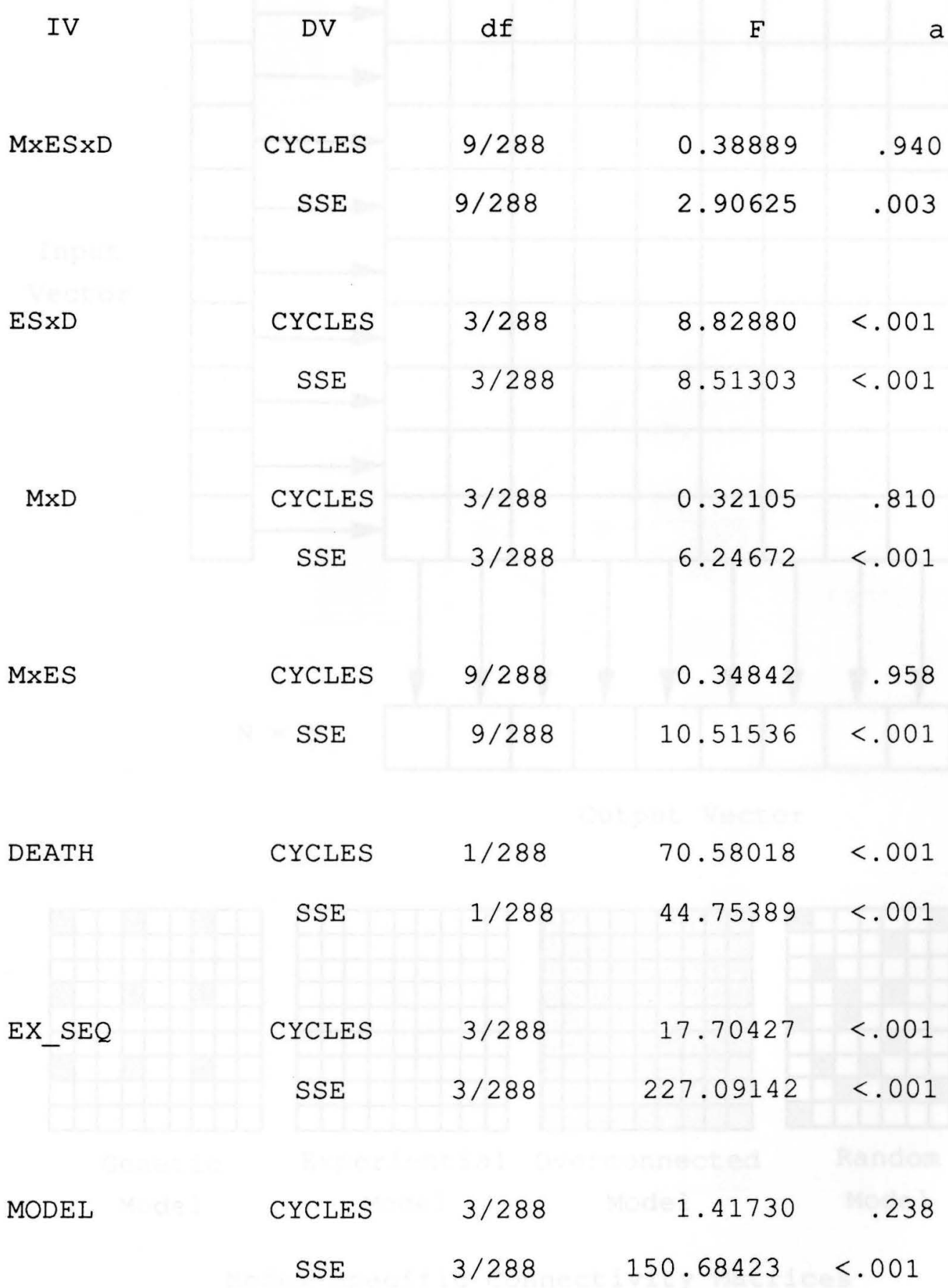




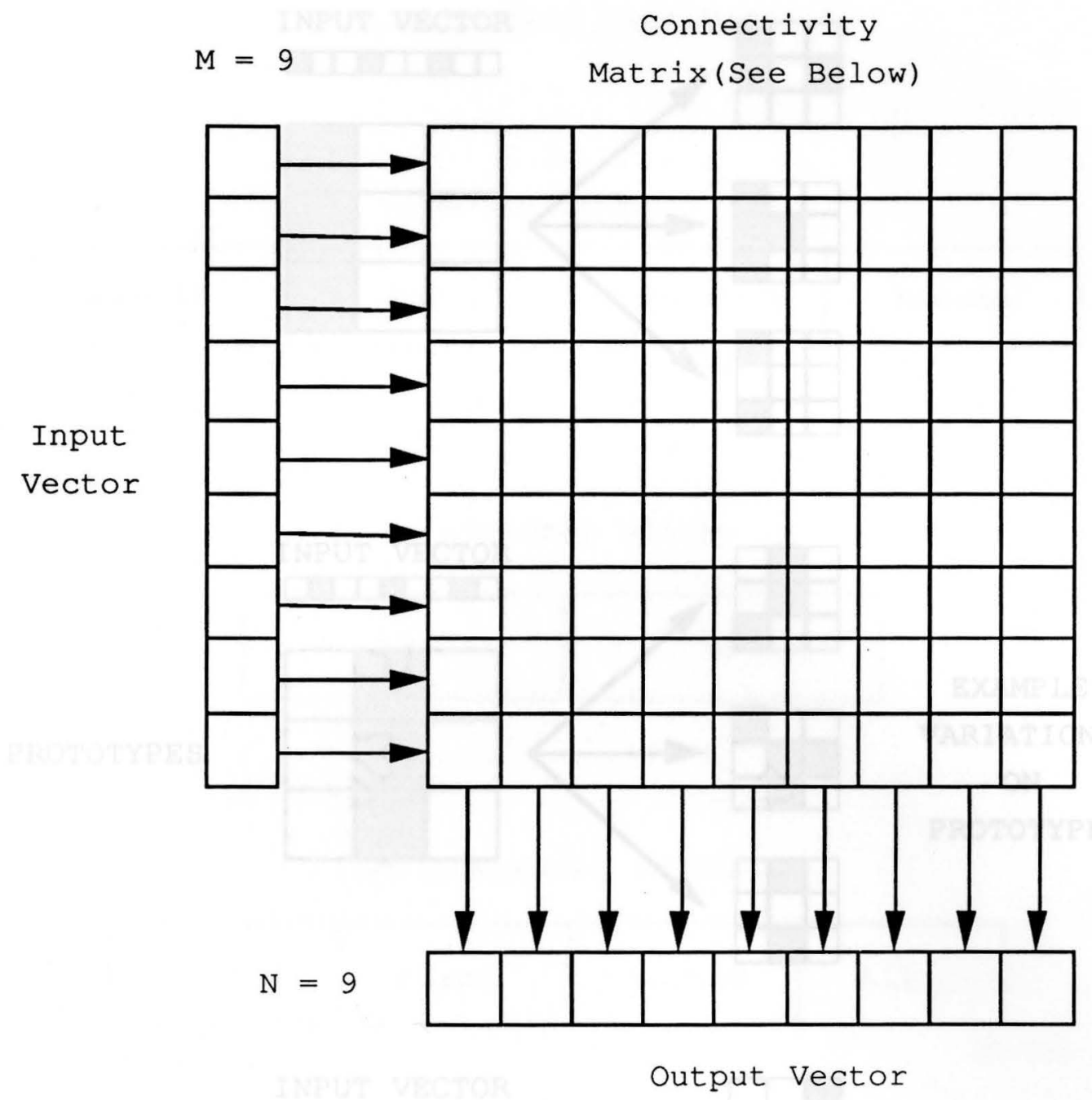

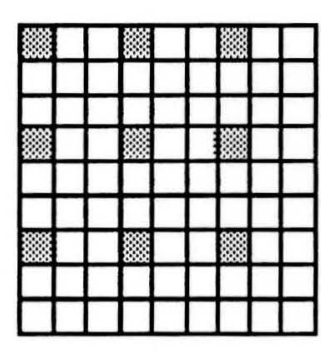

Genetic

Mode 1

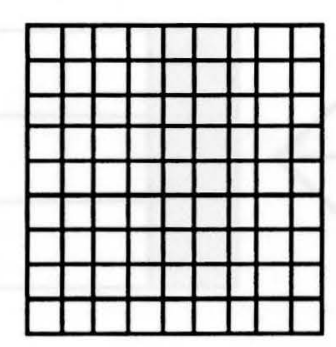

Experiential Model

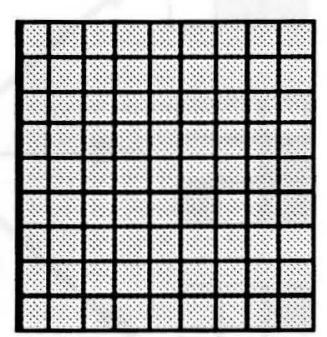

Overconnected

Mode 1

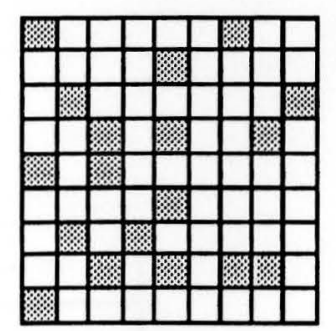

Random

Model

Model-Specific Connectivity Matrices

Figure 1. Overall Network Architecture 

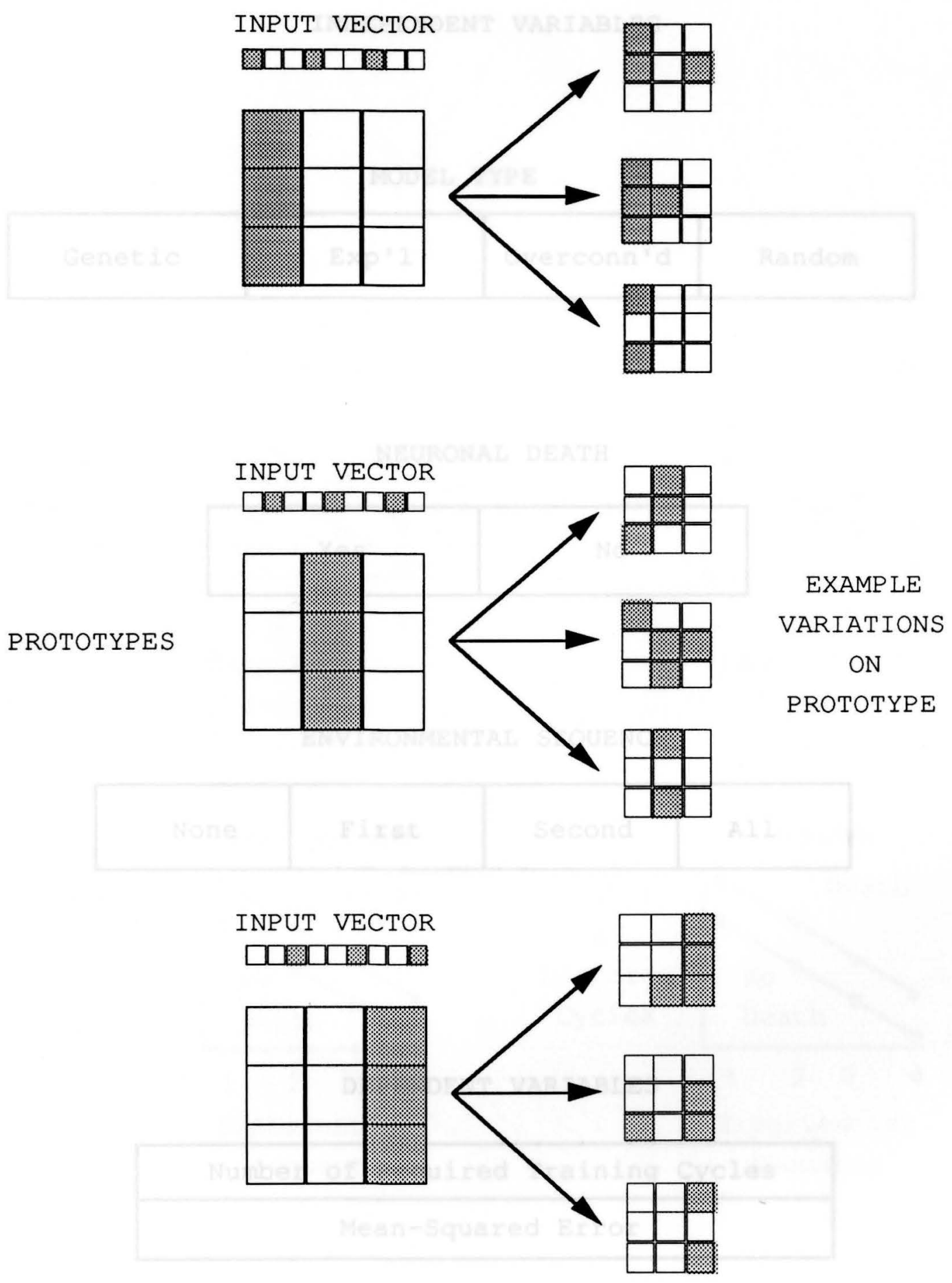

Figure 2. Prototype and Test Stimuli 
MODEL TYPE

\begin{tabular}{|l|l|l|l|}
\hline Genetic & Exp'l & Overconn'd & Random \\
\hline
\end{tabular}

NEURONAL DEATH

\begin{tabular}{|l|l|}
\hline Yes & No \\
\hline
\end{tabular}

ENVIRONMENTAL SEQUENCE

\begin{tabular}{|l|l|l|l|}
\hline None & First & Second & All \\
\hline
\end{tabular}

DEPENDENT VARIABLES

Number of Required Training Cycles

Mean-Squared Error

Figure 3. Experimental Design 
Genetic

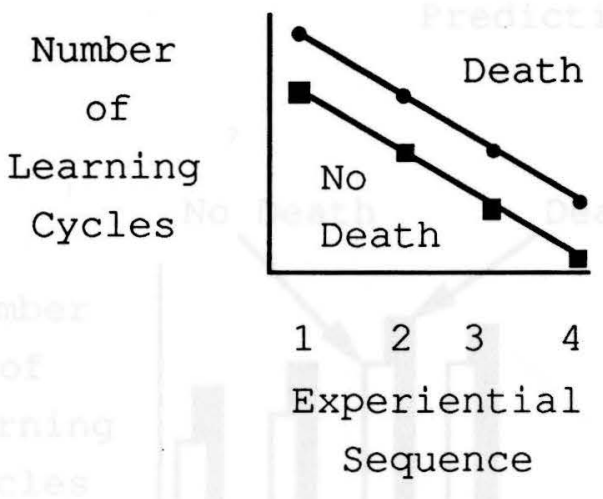

Experiential

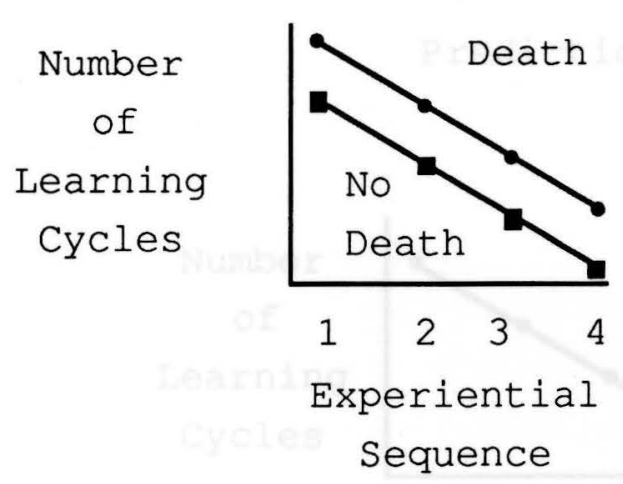

Overconnected

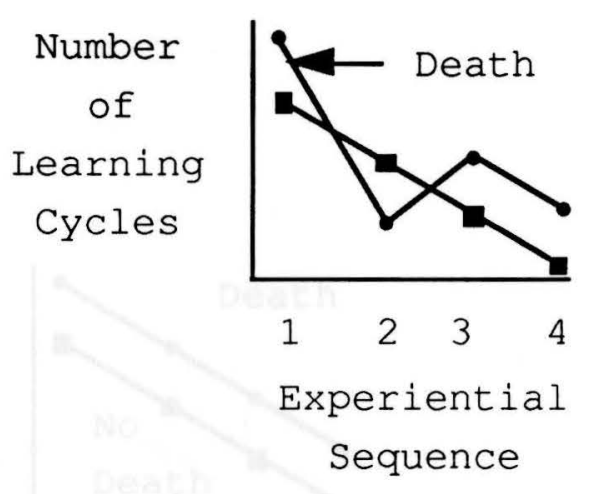

Random
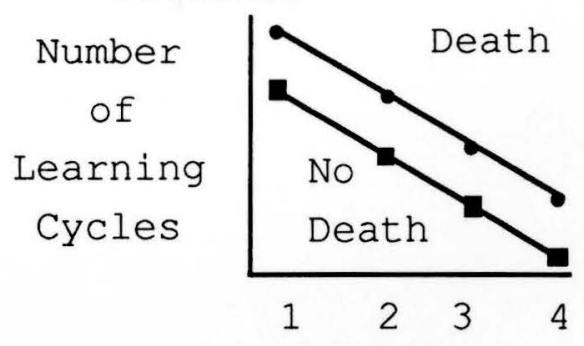

Experiential sequence

Prediction 1

Figure 4a. Predicted Effects 

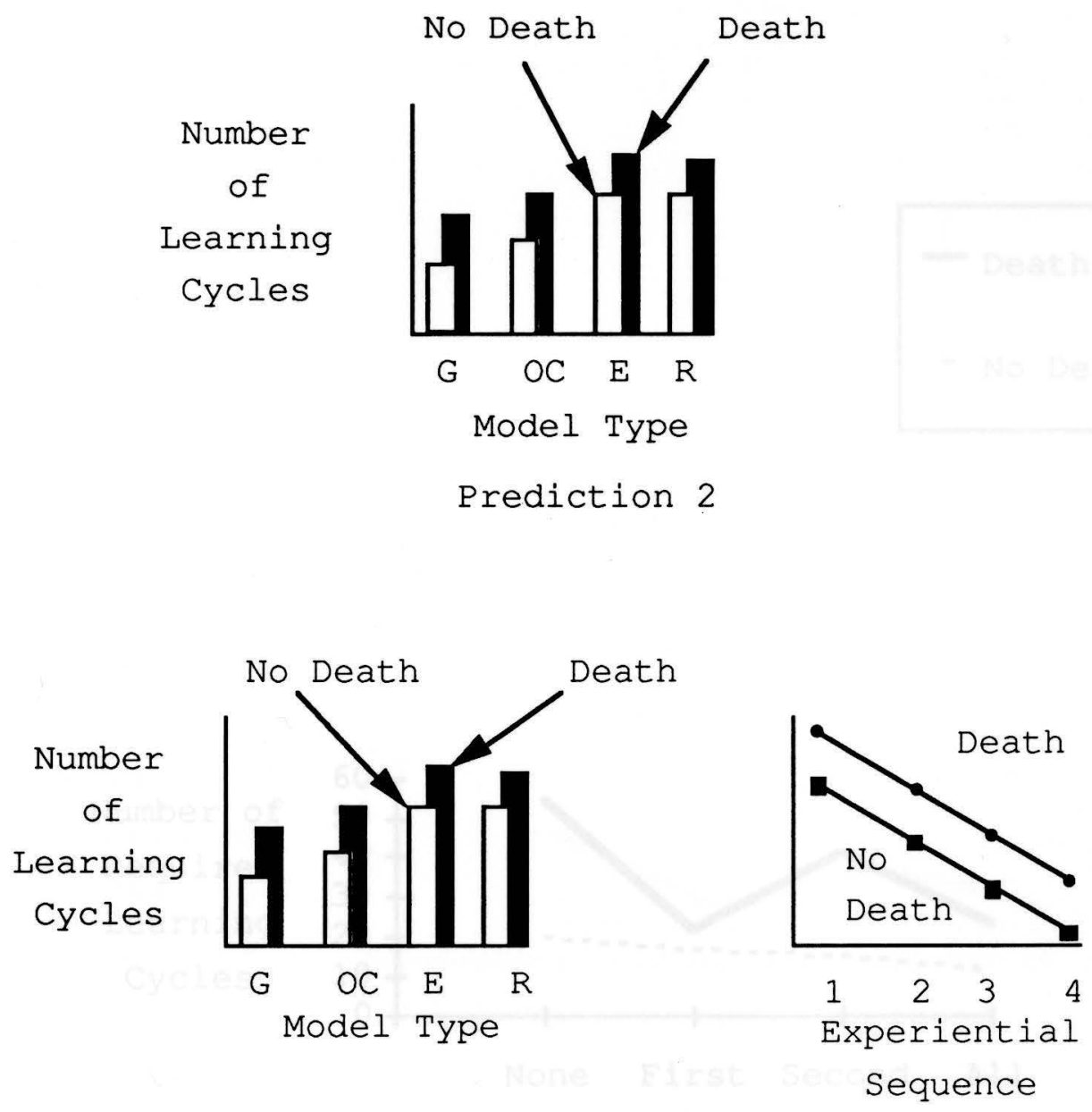

Prediction 3

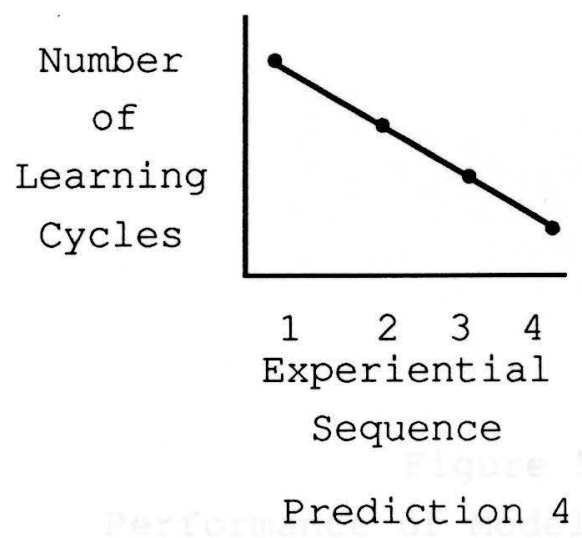

Figure 4b. Predicted Effects 


$$
\begin{aligned}
& \text { - Death } \\
& \text { - No Death }
\end{aligned}
$$

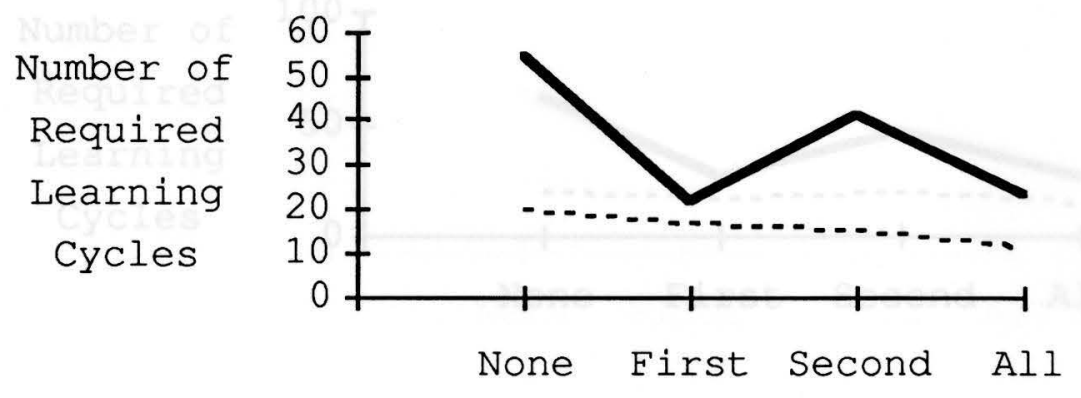

Variant Stimuli Exposure Sequence

Figure 5.

Performance of Model as a Function

of Environmental Sequence 


\section{Death \\ - No Death}

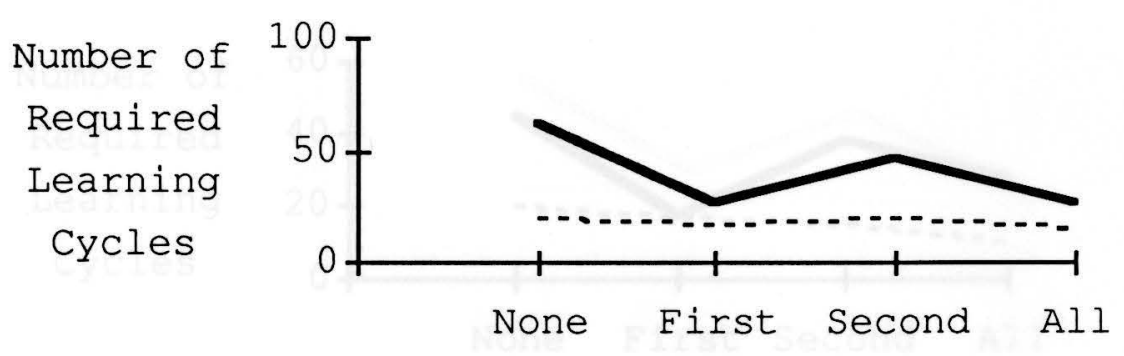

Variant Stimuli Exposure Sequence

Figure $6 a$.

Genetic Model's Ability to Learn as a

Function of Environmental Sequence 


$$
\begin{aligned}
& \text { - Death } \\
& \text {-- No Death }
\end{aligned}
$$

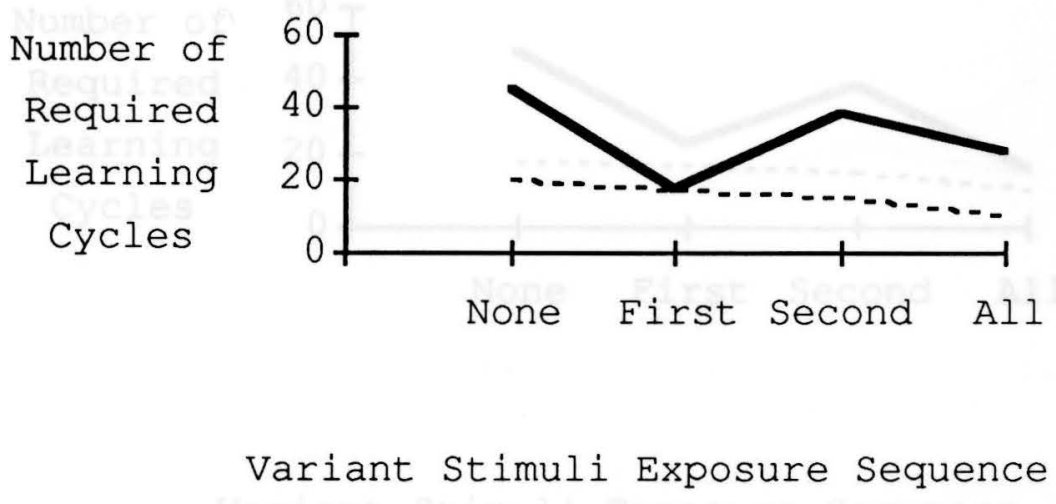

Eigure $6 \mathrm{~b}$.

Experiential Model's Ability to Learn as a

Function of Environmental Sequence 

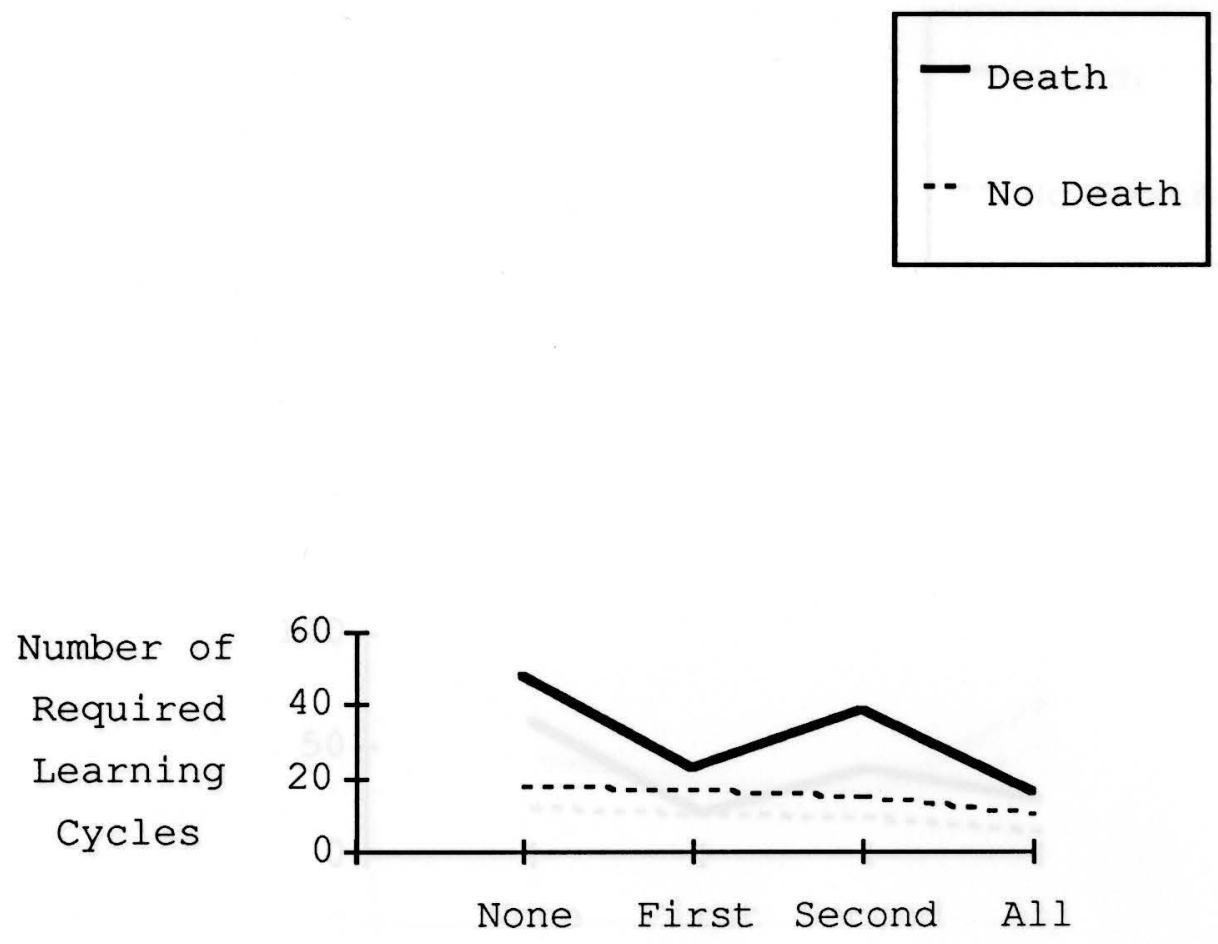

Variant Stimuli Exposure Sequence

Figure 6c.

Overconnected Model's Ability to Learn as a

Function of Environmental Sequence 


$$
\begin{aligned}
& \text { - Death } \\
& \text {-- No Death }
\end{aligned}
$$

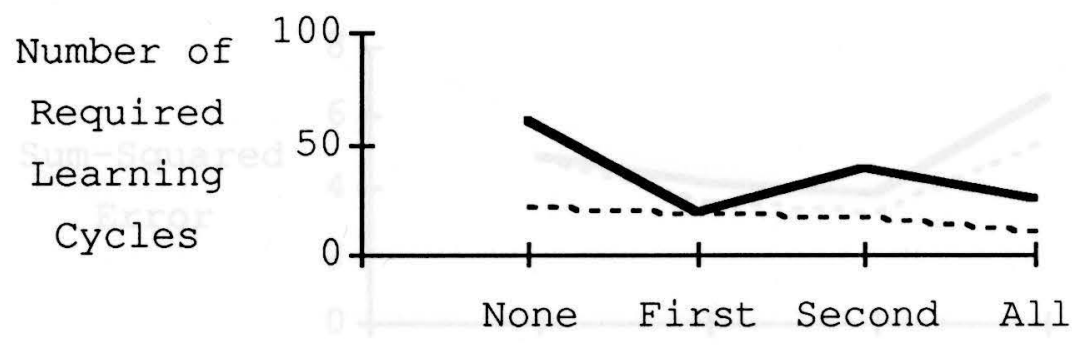

Variant Stimuli Exposure Sequence

Figure 6d.

Random Model's Ability to Learn as a Function of Environmental Sequence 

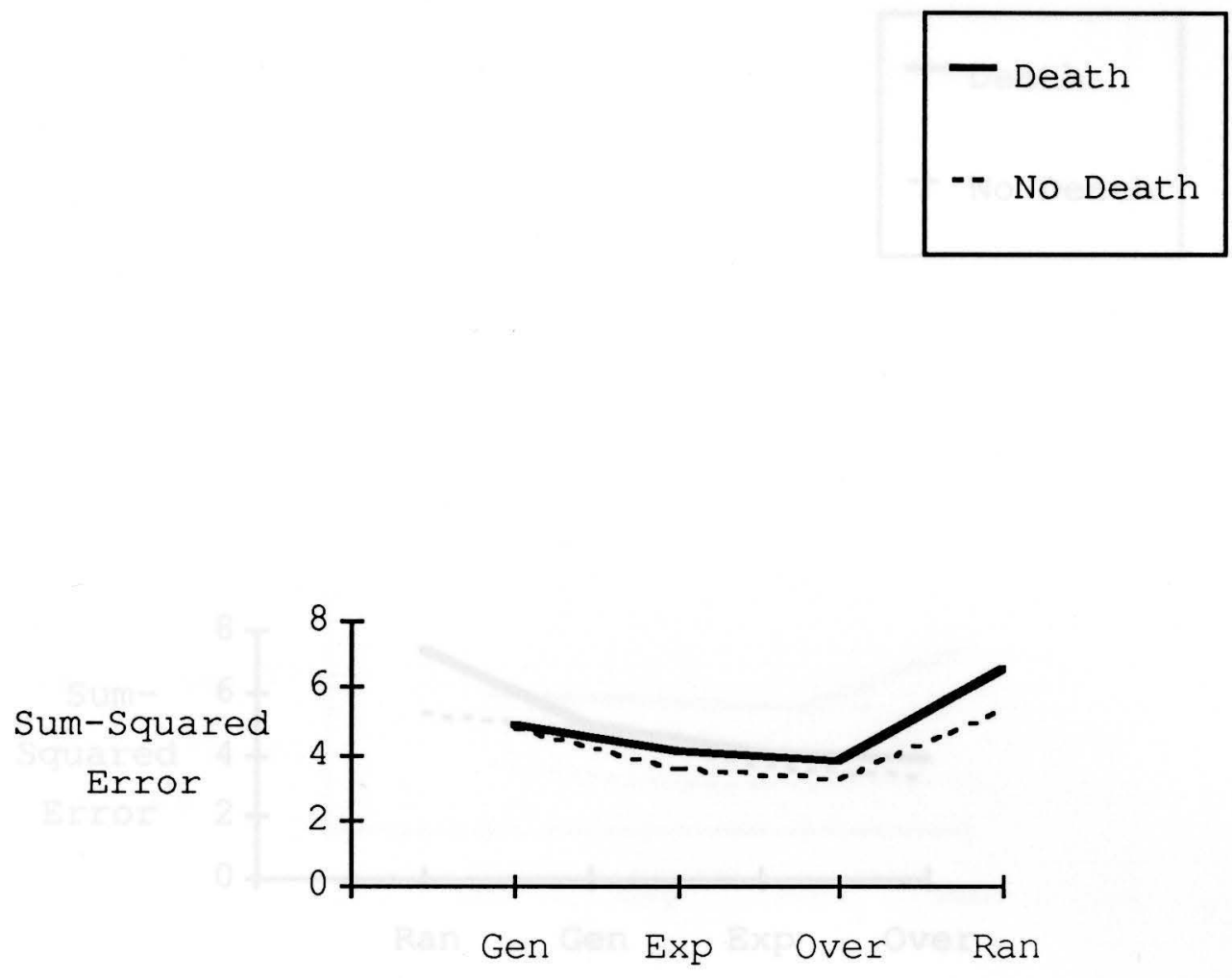

Model Type

Figure 7a.

Structural Adaptation as a Function

of Model Type 

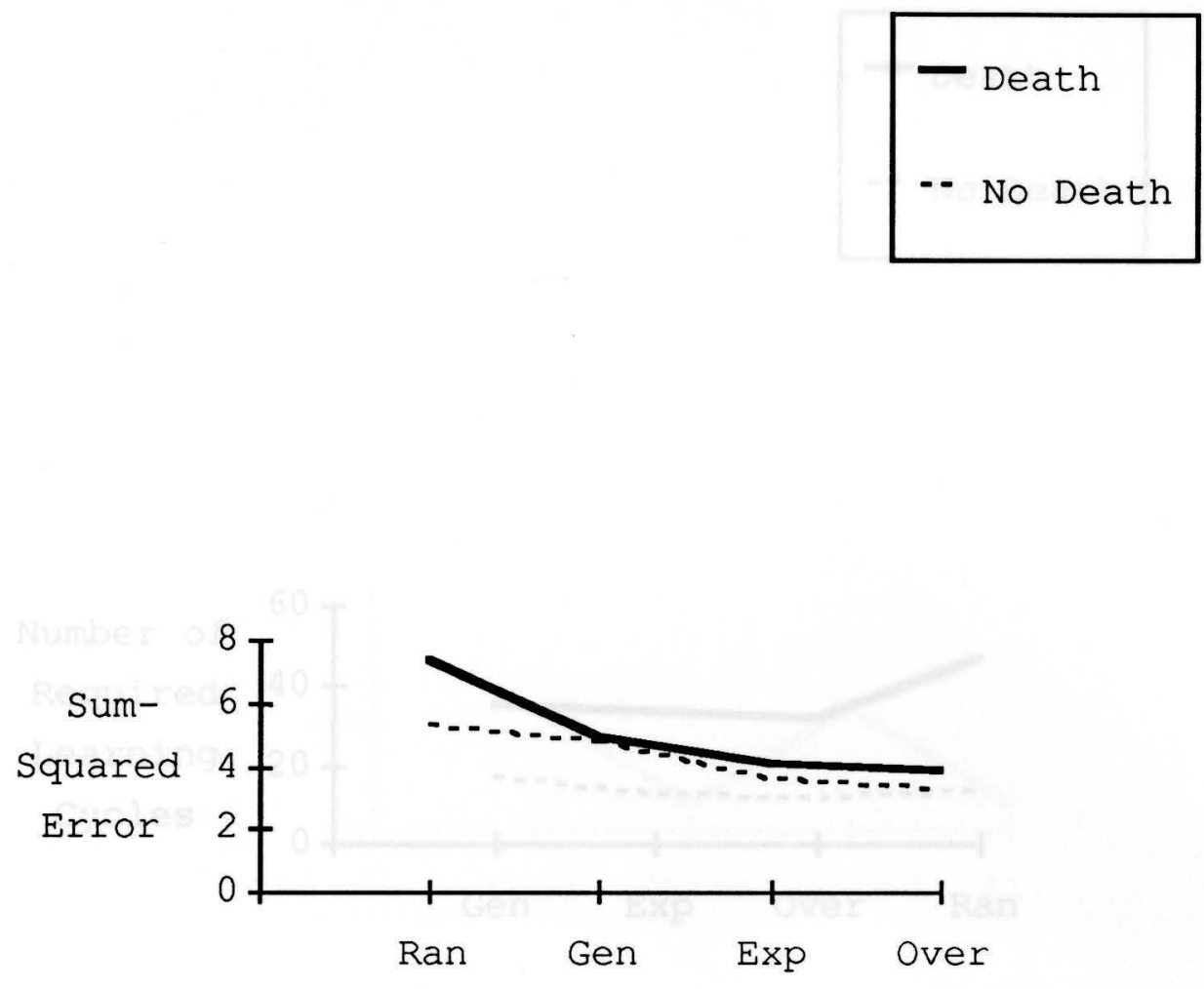

Model Type

Figure 7b.

Structural Adaptation as a Function

of Model Type (by Increasing Error) 

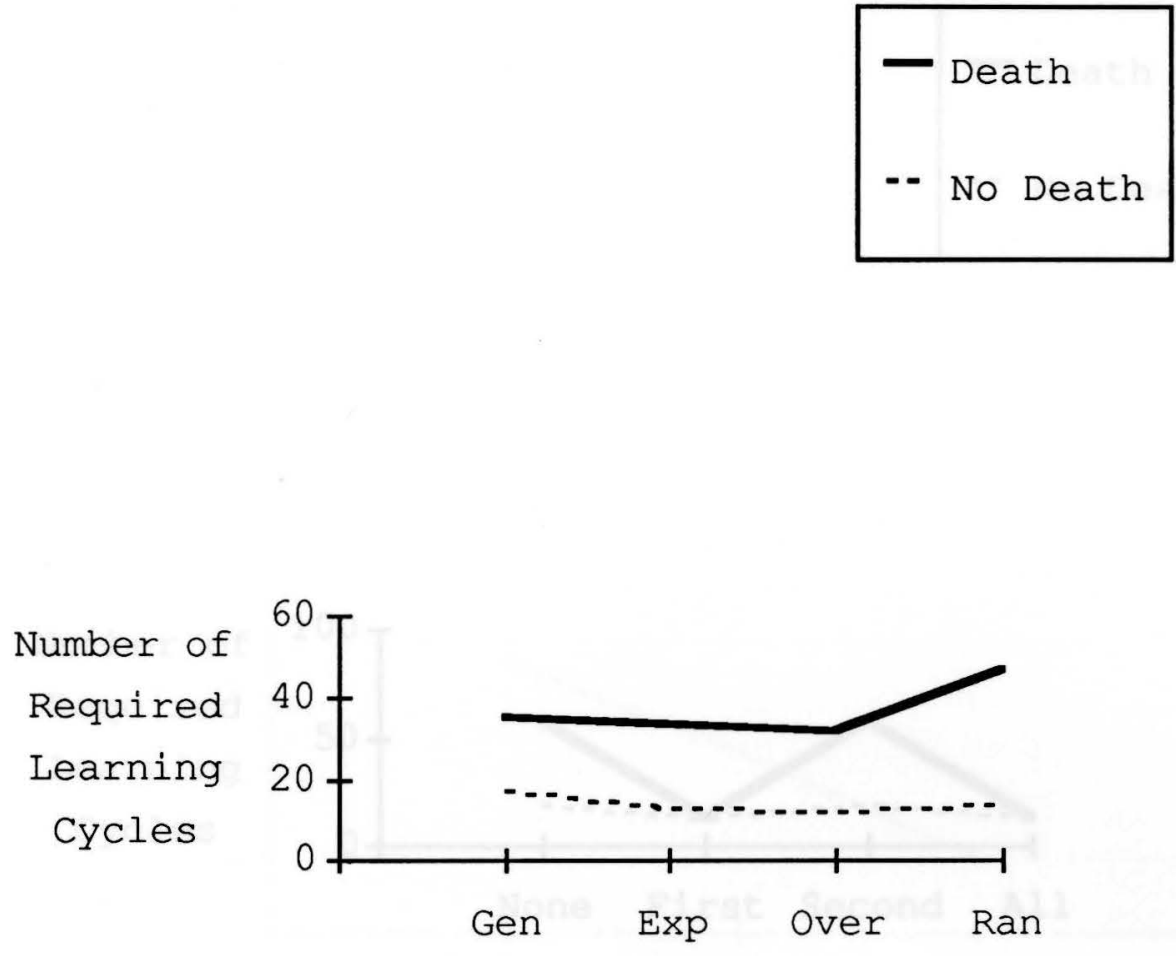

Model Type

Figure 8 .

Structural Adaptation as a Function

of Model Type (No Noise in Training Set) 

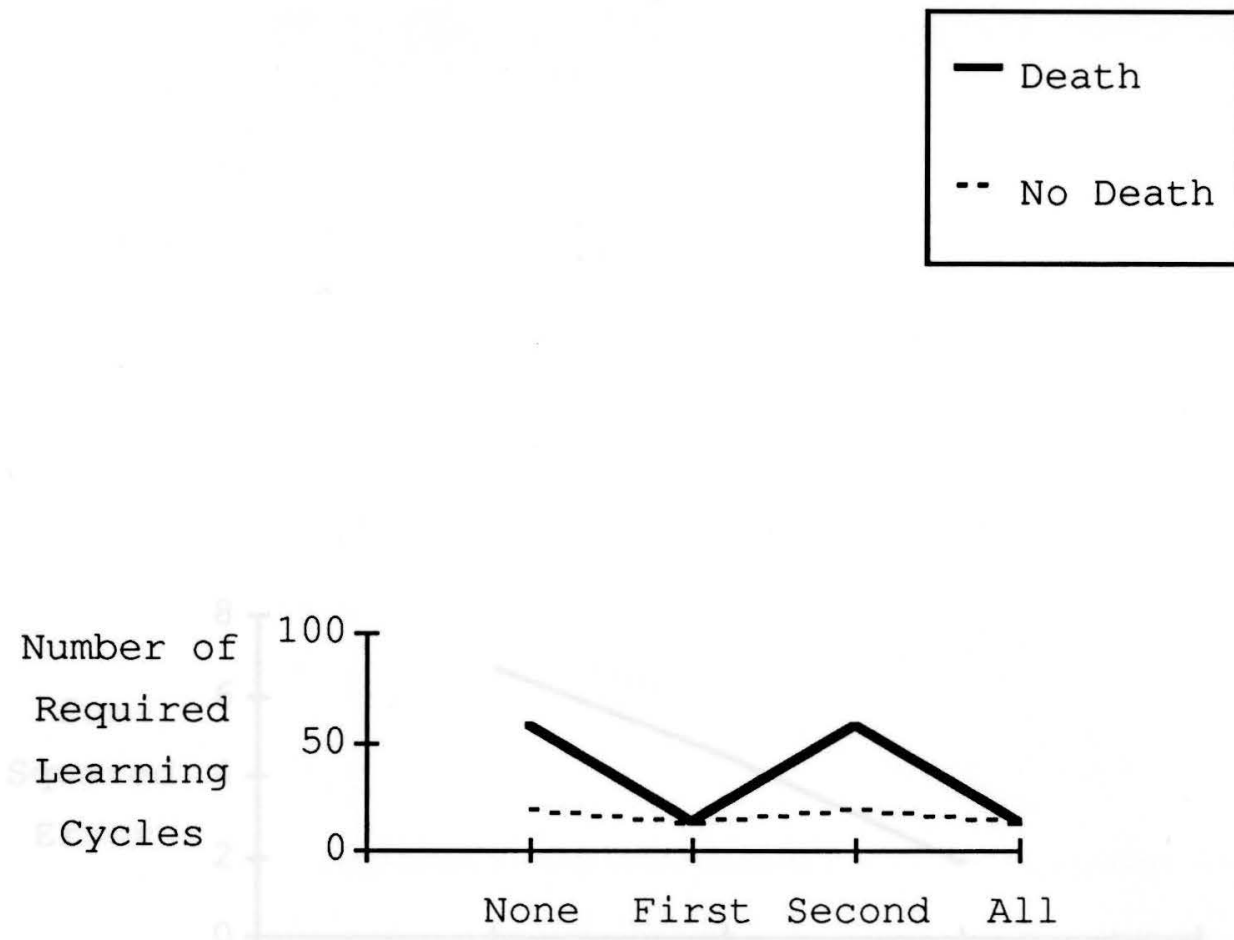

Variant Stimuli Exposure Sequence

Figure 9.

Genetic Model's Performance as a Function of Environmental Sequence (No Noise in Training Set) 


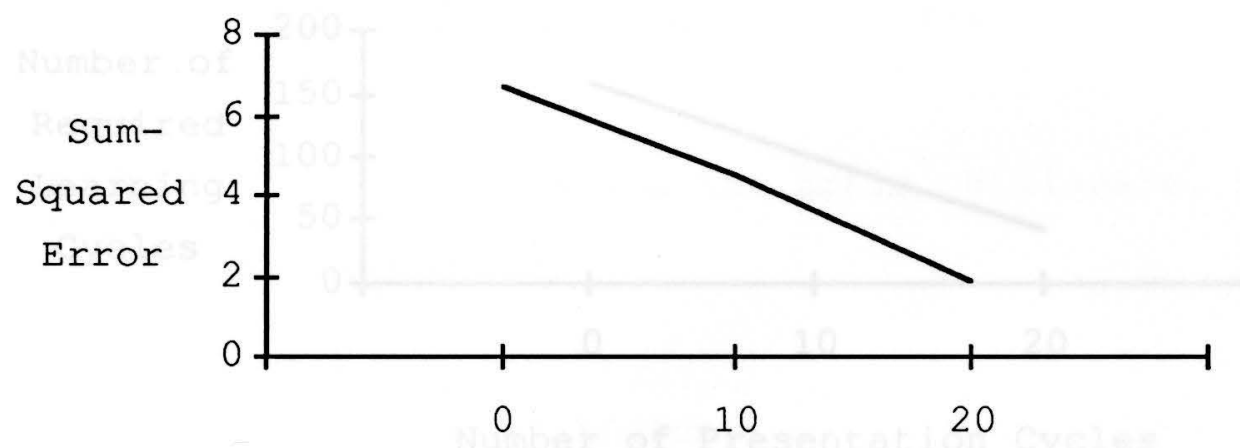

Number of Presentation Cycles

Figure 10a.

Structural Fit of Models as a Function

of Amount of Stimulus Exposure 


\section{BIBLIOGRAPHY}

Alkon, D.L. (1987). Memory Traces in the Brain. Cambridge: Cambridge University Press.

Alkon, D.L. (1989). "Memory Storage and Neural Systems." Scientific American, 261, 42-50.

Aoki, C. and Siekevitz, P. (1988). "Plasticity in Brain Development." Scientific American, 259, 56-64.

Arnold, M.B. (1984). Memory and the Brain. Hillsdale, NJ: Lawrence Erlbaum Associates.

Bandura, A. (1986). Social Foundations of Thought and Action. Englewood Cliffs, NJ: Prentice-Hall, Inc.

Black, I.B., et.al (1984). "Neurotransmitter Plasticity at the Molecular Level." Science. 225, 1266-1270.

Bolles, R.C. (1979). Learning Theory. Holt, Rinehart and Winston.

Bornstein, M.H. and Lamb, M.E. (1984). Developmental Psychology: An Advanced Textbook. Hillsdale, NJ: Lawrence Erlbaum Associates. 
Brown, T.H., Chapman, P.F., Kairiss, E.W. and Keenan, C.L. (1988) . "Long-Term Synaptic Potentiation." Science, 242, 724728.

Byrne, J.H. and Berry, W.O. (1989). Neural Models of

Plasticity, San Diego, CA: Academic Press.

Catania, A.C. (1984). Learning. Englewood Cliffs, NJ:

Prentice -Hall, Inc.

Carlson, N.R. (1986). Physiology of Behavior. Boston, MA: Allyn and Bacon, Inc.

Caudill, M. and Butler, C. (1990). Naturally Intelligent Systems, Cambridge, MA: MIT Press.

Coleman, J.C., Butcher, J.N., and Carson, R.C. ((1980). Abnormal Psychology and Modern Life. Glenview, IL: Scott, Foresman and Company.

Collyer, C.E. (1985). "Comparing strong and weak models by fitting them to computer-generated data." Perception \& Psychphysics, 38, (5), 476-481.

Collyer, C.E. (1986). "Goodness-of-fit patterns in a computer cross-validation procedure comparing a linear and a threshold 
model." Behavior Research Methods, Instruments, \& Computers, $18,(6), 618-622$.

Collyer, C.E. (1988). "Parameter Estimates depend both on the source model and the fitted model: An example." Bulletin of the Psychometric Society, 26, (4), 289-292.

Cotman, C.W. and Nieto-Sampedro, M. (1984). "Cell Biology of Synaptic Plasticity." Science, 225, 1287-1294.

Elkind, D.E. and Flavell, J.H. (1969). Studies in Cognitive Development. New York: Oxford University Press.

Fallman, W.F. (1989). Apprentices of Wonder. New York: Bantam Books.

Flavell, J.H. (1985). Cognitive Development. Englewood Cliffs, NJ: Prentice-Hall, Inc.

Gardner, H. (1986). The Mind's New Science. New York: Basic Books, Inc.

Gazzaniga, M.S. (1985). The Social Brain. New York: Basic Books, Inc. 
Hawkins, R.D. and Bopwer, G.H. (1989). Computational Models of Learning in Simple Neural Systems. San Diego, CA: Academic Press.

Hinton, G.E. and Anderson, J.A. (1981). Parallel Models of Associative Memory. Hillsdale, NJ: Lawrence Erlbaum Associates.

Jaynes, J. (1976). The origin of consciousness in the Breakdown of the Bicameral Mind. Boston, MA: Houghton Mifflin Company .

Kalil, R.E. (1989). "Synapse Formation in the Developing Brain." Scientific American, 261, 76-85.

Kanerva, P. (1988). Sparse Distributed Memory. Cambrdige, MA: MIT Press.

Knapp, A.G. and Anderson, J.A. (1984). "Theory of Categorization Based on Distributed Memory Storage." Journal of Experimental Psychology: Learning, Memory and Cognition, $10,616-637$.

Lerner, R.M. (1986). Concepts and Theories of Human Development. New York: Random House. 
Lichtman, J.W. (1977). "The Reorganization of Synaptic Connections in the Rat Submandibular Ganglion During PostNatal Development." J.Physiol,, 273, 155-177.

Macleod, R.B. (1975). The Persistent Problems of Psychology. Pittsburgh, PA: Duquesne Press.

Maturana, H.R. and Varela, F.J. (1987). The Tree of Knowledge, Boston, MA: Shambhala.

McClelland, J.L. and Rumelhart, D.E. (1985). "Distributed Memory and the Representation of General and Specific Information." Journal of Experimental Psychology, 114, 159188.

McCrone, J. (1991). The Ape That Spoke, New York: William Morrow and Company.

Minsky, M. (1986). The Society of Mind. New York: Simon and Schuster.

Meyer, D.R. and Meyer, P.M. (1977). "Dynamics and bases of recoveries of functions after injuries to the cerebral cortex." Physiological Psychology. Vol.5 (2), 133-165.

Munro, P.W. (1986). "State-Dependent Factors Influencing Neural Plasticity: A Partial Account of the Critical Period." 
In J.L. MCClelland and D.E. Rumelhart (Eds.) Parallel Distributed Processing. Cambridge, MA: Bradford Books / MIT Press.

Purves, D. and Lichtman, J.W. (1980). "Elimination of Synapses in the Developing Nervous System." Science, 210, $153-157$.

Rakic, P. and Riley, K.P. (1983). "Overproduction and Elimination of Retinal Axons in the Fetal Rhesus Monkey." Science, 219, 1441-1444.

Restak, R.M. (1988). The Mind. New York: Bantam Books.

Scott, J.P. (1962). "Critical Periods in Behavioral Development." Science, 138, 949-957.

Schwartz, B. (1989). Psychology of Learning and Behavior. New York: W.W. Norton and Company.

Shepherd, G.M. (1990). The Synaptic Organition of the Brain. New York: Oxford University Press.

Stillings, N.A., et. al (1989). Cognitive Science, Cambridge, MA: MIT Press. 
Squire, L.R. (1987). Memory and Brain. New York: Oxford University Press.

Spinelli, D.N., and Jensen, F.E. (1978). "Plasticity: The Mirror of Experience." Science, 203, 75-78.

Springer, S.P. and Deutsch, G. (1985). Left Brain and Right Brain. New York: W.H. Freeman and Company.

Tabachnick, B.G., and Fidell, L.S. (1983). Using Multivariate Statistics. New York: Harper Row.

Zuriff, G.E. (1985). Behaviorism: A Conceptual

Reconstruction. New York: Columbia University Press. 